OPEN ACCESS

Edited by:

Jianrong Steve Zhou,

Shenzhen Graduate School, Peking

University, China

Reviewed by:

Xiaolei Huang,

Zhejiang Normal University, China

Chunquan Sheng,

Second Military Medical University,

China

*Correspondence:

Yong-Xian Cheng

yxcheng@szu.edu.cn

Li-Zhong Liu

liulz@szu.edu.cn

Specialty section:

This article was submitted to

Organic Chemistry,

a section of the journal

Frontiers in Chemistry

Received: 26 September 2021

Accepted: 13 October 2021

Published: 03 December 2021

Citation:

Qin F-Y, XU T, Li Y-P, Zhang H-X,

Cai D, Liu L-Z and Cheng Y-X (2021)

Terminal Cyclohexane-Type

Meroterpenoids from the Fruiting

Bodies of Ganoderma cochlear.

Front. Chem. 9:783705.

doi: 10.3389/fchem.2021.783705

\section{Terminal Cyclohexane-Type Meroterpenoids from the Fruiting Bodies of Ganoderma cochlear}

\author{
Fu-Ying Qin ${ }^{1}$, Te Xu ${ }^{1}$, Yan-Peng $L^{1}{ }^{1}$, Hao-Xing Zhang ${ }^{1}$, Dan Cai ${ }^{1}$, Li-Zhong Liu ${ }^{1 *}$ and \\ Yong-Xian Cheng ${ }^{1,2 *}$
}

${ }^{1}$ School of Pharmaceutical Sciences, School of Medicine, College of Life Sciences and Oceanography, Health Science Center, Institute for Inheritance-Based Innovation of Chinese Medicine, Shenzhen University, Shenzhen, China, ${ }^{2}$ Guangdong Key Laboratory for Functional Substances in Medicinal Edible Resources and Healthcare Products, School of Life Sciences and Food Engineering, Hanshan Normal University, Chaozhou, China

Eleven new cyclohexane-type meroterpenoids (1, 3-5, 7, 8, 11-15) and four known similar meroterpenoids $(\mathbf{2}, \mathbf{6}, \mathbf{9}$, and $\mathbf{1 0})$ were isolated from Ganoderma cochlear. Their structures and absolute configurations at stereogenic centers were elucidated by using HRESIMS, NMR spectroscopy and computational methods. In addition, the structure of the known meroterpenoid, cochlearol G (2), was revised, and the absolute configurations at the stereogenic centers of known meroterpenoids $\mathbf{9}$ and $\mathbf{1 0}$ were determined. All the isolated meroterpenoids were evaluated for their activities against renal fibrosis and triple negative breast cancer, and their insulin resistance. The results of the renal fibrosis study showed that meroterpenoid $\mathbf{1 1}$ inhibits over-expression of fibronectin, collagen I and $\alpha$-SMA. Results of the wound healing study revealed that $\mathbf{4 , 6}$ and $\mathbf{8}$ significantly inhibit migration of BT549 cells. Observations made in Western blotting experiments showed that $\mathbf{6}$ decreases the levels of TWIST1 and ZEB1, and increases the level of E-cadherin. Finally, meroterpenoids $\mathbf{7}, \mathbf{9}, \mathbf{1 1}$, and $\mathbf{1 5}$ significantly up-regulate p-AMPK protein expression in normal L6 myotubes cells.

Keywords: Ganoderma cochlear, meroterpenoids, renal fibrosis, triple negative breast cancer, BT549 cells

\section{INTRODUCTION}

Ganoderma is not only a famous Chinese medicine, it is also used globally as a food, in the form of tea, coffee and other beverages, and in syrups and dietary supplements (Wang et al., 2020; Kumar, 2021). Polysaccharides and triterpenoids are representative of the important biologically active components of Ganoderma (Wang et al., 2020). Recent ongoing research studies exploring Ganoderma demonstrated that it contains meroterpenoid components that possess extensive biological activities, such as renal protection and neuroprotection, anti-inflammation, -tumor and -oxidation properties, and analgesic effects (Jiang et al., 2021). These studies led to a deeper understanding of the components of Ganoderma and insight into active ingredients responsible for its traditional medical properties.

Ganoderma meroterpenoids comprise a class of substances with great potential, not only because they contain a variety of structural subtypes, but also because many members possess a host of biological activities. For instance, in 2009 ganomycin I was found to inhibit HIV-1 protease, and then in 2014 it was shown to have inhibitory effects on the production of monocyte chemotactic protein 1 (MCP-1) and fibronectin. Following these discoveries, ganomycin I was 
observed to inhibit NSC proliferation in 2015, and then in 2017 it was discovered to display hypoglycemic, hypolipidemic and insulin-sensitizing effects (Dine et al., 2009; Luo et al., 2015; Yan et al., 2015; Wang et al., 2017). As a result, we have been engaged in a program to isolate and identify new Ganoderma meroterpenoids and to assess their unique biological activities. In a previous effort, we found that these substances have inhibitory effects on renal fibrosis (Luo et al., 2015; Meng et al., 2021). In the current investigation, we isolated fifteen terminal cyclohexane-type meroterpenoids (1-15) from Ganoderma cochlear and evaluated their biological activities against renal fibrosis and insulin resistance. Breast cancer is one of the malignant cancer, and the morbidity and mortality is highest in women, with triple negative breast cancer (TNBC) being. Based on the fact that Ganoderma has been used to treat cancer, we also investigated the activities of the meroterpenoids against cells of triple negative breast cancer, which is difficult to treat cancers and has an extremely high mortality rate (Collignon et al., 2016). The results of this study are described below.

\section{MATERIALS AND METHODS}

\section{General}

Optical rotations were determined using an Anton Paar MCP100 digital polarimeter. $\mathrm{UV}$ and $\mathrm{CD}$ spectra were recorded on a Chirascan instrument. NMR spectra were obtained by using a Bruker Avance III $600 \mathrm{MHz}$ or $500 \mathrm{MHz}$ spectrometer, with TMS as an internal standard. All NMR chemical shifts are given in ppm. HRESIMS were recorded using a Shimazu LC20AD AB SCIEX triple TOF 6600+ MS spectrometer (Shimadzu Corporation, Tokyo, Japan). MCI gel CHP 20P (75-150 $\mu \mathrm{m}$, Mitsubishi Chemical Industries, Tokyo, Japan), C-18 silica gel (40-60 $\mu \mathrm{m}$; Daiso Co., Japan), and Sephadex LH-20 (Amersham Pharmacia, Uppsala, Sweden) were used for column chromatography. Silica gel (Qingdao Marine Chemical Inc., Qingdao, China) was used for vacuum column chromatography (VLC). Preparative HPLC was carried out using a Saipuruisi chromatograph equipped with a Thermo Hypersil GOLD-C18 column $(250 \times 21.2 \mathrm{~mm}$, i. d., $5 \mu \mathrm{m})$. Semi-preparative HPLC was carried out using a Saipuruisi chromatograph with a YMC-Pack ODS-A column $(250 \times 10 \mathrm{~mm}$, i. d., $5 \mu \mathrm{m})$. Chiral HPLC analysis was run using an Agilent 1260 chromatograph with a Daicel Chiralpak column (IC, $250 \mathrm{~mm} \times 10 \mathrm{~mm}$, i. d., $5 \mu \mathrm{m}$ ) or a Daicel Chiralpak column (IC, $250 \mathrm{~mm} \times 4.6 \mathrm{~mm}$, i. d., $5 \mu \mathrm{m}$ ).

\section{Fungal Material}

The dried fruiting bodies of G. cochlear were purchased from Tongkang Pharmaceutical Co. Ltd. Guangdong province, China, in July 2014. This fungus was authenticated by Prof. Zhu-Liang Yang at Kunming Institute of Botany, Chinese Academy of Sciences, China, and a voucher specimen (CHYX-0589) is deposited at Institute for Inheritance-Based Innovation of Chinese Medicine, Shenzhen University Health Science Center, China.

\section{Extraction and Isolation}

Powders of $G$. cochlear $(200 \mathrm{~kg})$ fruiting bodies were extracted with refluxing $80 \% \mathrm{EtOH}(3 \times 120 \mathrm{~L}, 4,3,3 \mathrm{~h})$ and the extract was concentrated under reduced pressure to afford a crude residue. An aliquot ( $8 \mathrm{~kg}$ of the residue corresponding to $95 \mathrm{~kg}$ fungal material) was suspended in water and extracted three times with EtOAc, followed by concentration of the combined extracts to afford an EtOAc soluble residue $(4 \mathrm{~kg}$ ). The residue was subjected to silica gel column using an eluant comprised of increasing amounts of acetone in petroleum ether to provide four parts (Fr.1-Fr.4). Fr.2 (860 g) was further divided into six parts (Fr.2.1-Fr.2.6) by MCI gel CHP 20P ( $\left.\mathrm{MeOH} / \mathrm{H}_{2} \mathrm{O}, 60-100 \%\right)$. Fr.2.2 (120.0 g) was divided into five parts (Fr.2.2.1-Fr.2.2.5) by using RP-18 column chromatography (eluting solvent: $\mathrm{MeOH} /$ $\left.\mathrm{H}_{2} \mathrm{O}, 40-100 \%\right)$. Fr.2.2.3 (21.0 g) was subjected to a MCI gel CHP $20 \mathrm{P}$ column chromatography $\left(\mathrm{MeOH} / \mathrm{H}_{2} \mathrm{O}, 40-100 \%\right)$ to obtain five parts (Fr.2.2.3.1-Fr.2.2.3.5). Fr.2.2.3.3 (3.7 g) was gel filtered through Sephadex $\mathrm{LH}-20(\mathrm{MeOH})$ to produce three parts (Fr.2.2.3.3.1-Fr.2.2.3.3.3.). Fr.2.2.3.3.1 (1.9 g) was subjected to preparative $\mathrm{HPLC}\left(\mathrm{MeOH} / \mathrm{H}_{2} \mathrm{O}, 65-100 \%\right)$ to produce three parts (Fr.2.2.3.3.1.1-Fr.2.2.3.3.1.3). Semi-preparative HPLC (eluting solvent: $\mathrm{MeOH} / \mathrm{H}_{2} \mathrm{O}$ containing $0.05 \%$ TFA, $60-100 \%$, flow rate: $3 \mathrm{~mL} / \mathrm{min})$ of Fr.2.2.3.3.1.2 (0.9 g) gave three subfractions (Fr.2.2.3.3.1.2.1-Fr.2.2.3.3.1.2.3). Semi-preparative HPLC (eluting solvent: $\mathrm{MeOH} / \mathrm{H}_{2} \mathrm{O}$ containing $0.05 \%$ TFA, $70 \%$, flow rate: $3 \mathrm{~mL} / \mathrm{min}$ ) of subfraction Fr.2.2.3.3.1.2.3 (300 mg) yielded 12 (5.6 mg, $\left.t_{R}=20.5 \mathrm{~min}\right)$. Semi-preparative HPLC (eluting solvent: $\mathrm{MeOH} / \mathrm{H}_{2} \mathrm{O}$ containing $0.05 \% \mathrm{TFA}, 65 \%$, flow rate: $3 \mathrm{~mL} / \mathrm{min}$ ) of Fr.2.2.3.3.1.3 (200 mg) produced subfraction Fr.2.2.3.3.1.3.3 (13.1 $\left.\mathrm{mg}, \mathrm{t}_{\mathrm{R}}=20.7 \mathrm{~min}\right)$, which by using chiral Daicel Chialpak IC column (n-hexane/ethanol containing $0.05 \%$ TFA, 92:8, flow rate: $1 \mathrm{~mL} / \mathrm{min}$ ) yielded $\mathbf{1 4}$ $\left(4.0 \mathrm{mg}, \mathrm{t}_{\mathrm{R}}=13.9 \mathrm{~min}\right)$ and $15\left(5.9 \mathrm{mg}, \mathrm{t}_{\mathrm{R}}=20.0 \mathrm{~min}\right)$.

Fr.3 (780 g) was fractionated into eight parts (Fr.3.1-Fr.3.8) by using a MCI gel CHP 20P column chromatography $(\mathrm{MeOH} /$ $\left.\mathrm{H}_{2} \mathrm{O}, 40-100 \%\right)$. Fr.3.4 (120 g) was divided into three portions (Fr.3.4.1-Fr.3.4.3) by using RP-18 column chromatography $\left(\mathrm{MeOH} / \mathrm{H}_{2} \mathrm{O}, 20 \%-100 \%\right)$. Submission of Fr.3.4.1 (115 g) to a MCI gel CHP 20P column chromatography $\left(\mathrm{MeOH} / \mathrm{H}_{2} \mathrm{O}\right.$, 40-100\%) provided six portions (Fr.3.4.1.1-Fr.3.4.1.6). Fr.3.4.1.3 (21.6 g) was separated by using Sephadex LH-20 $(\mathrm{MeOH})$ into four parts (Fr.3.4.1.3.1-Fr.3.4.1.3.4). Fr.3.4.1.3.3 $(6.5 \mathrm{~g})$ was subjected to RP-18 $(50 \mu \mathrm{m})$ column chromatography to obtain five parts (Fr.3.4.1.3.3.1-Fr.3.4.1.3.3.5). Fr.3.4.1.3.3.1 (1.1 g) was gel filtered through Sephadex LH-20 $(\mathrm{MeOH})$ to produce two subfractions (Fr.3.4.1.3.3.1.1 and Fr.3.4.1.3.3.1.2). Fr.3.4.1.3.3.1.1 $(0.7 \mathrm{~g})$ was separated into three portions by using preparative TLC $\left(\mathrm{CH}_{2} \mathrm{Cl}_{2}: \mathrm{MeOH}=7: 1\right)$. Submission of Fr.3.4.1.3.3.1.1.2 $(\mathrm{Rf}=0.6$, $100 \mathrm{mg}$ ) to semi-preparative HPLC (acetonitrile: $\mathrm{MeOH}: \mathrm{H}_{2} \mathrm{O}$ containing $0.05 \%$ TFA, 25.5:25.5:49.0, flow rate: $3 \mathrm{~mL} / \mathrm{min}$ ) yielded $2\left(34.8 \mathrm{mg}, \mathrm{t}_{\mathrm{R}}=18.5 \mathrm{~min}\right)$ and $1\left(11.4 \mathrm{mg}, \mathrm{t}_{\mathrm{R}}=20.0 \mathrm{~min}\right)$. Subjection of Fr.3.4.1.3.3.1.1.3 ( $\mathrm{Rf}=0.8,200 \mathrm{mg}$ ) to chiral Daicel Chialpak IC column chromatography (n-hexane/ethanol containing $0.05 \%$ TFA, 91:9, flow rate: $3 \mathrm{~mL} / \mathrm{min})$ yielded $13\left(76.9 \mathrm{mg}, \mathrm{t}_{\mathrm{R}}=\right.$ $16.0 \mathrm{~min}$ ). Fr.3.4.1.3.3.3 (1.4 g) was filtered through Sephadex LH-20 $(\mathrm{MeOH})$ to produce four fractions (Fr.3.4.1.3.3.3.1-Fr.3.4.1.3.3.3.4). Fr.3.4.1.3.3.3.1 (79 mg) was subjected to semi-preparative HPLC 
( $\mathrm{MeOH}: \mathrm{H}_{2} \mathrm{O}$ containing $0.05 \%$ TFA, $62 \%$, flow rate: $3 \mathrm{~mL} / \mathrm{min}$ ) to yield $11\left(7.2 \mathrm{mg}, \mathrm{t}_{\mathrm{R}}=37.4 \mathrm{~min}\right)$. Fr.3.4.1.3.3.3.3 (0.8 g) was subjected to preparative HPLC (acetonitrile: $\mathrm{H}_{2} \mathrm{O}$ containing $0.05 \%$ TFA, $42 \%$, flow rate: $8 \mathrm{~mL} / \mathrm{min}$ ) to generate six fractions (Fr.3.4.1.3.3.3.3.1-Fr.3.4.1.3.3.3.3.6). Fr.3.4.1.3.3.3.3.2 (178.3 mg) was subjected to chiral Daicel Chiralpak IC column chromatography (n-hexane/isopropanol containing 0.05\% TFA, 91:9, flow rate: $3 \mathrm{~mL} / \mathrm{min})$ to yield $7\left(18.3 \mathrm{mg}, \mathrm{t}_{\mathrm{R}}=34.6 \mathrm{~min}\right)$ and $8\left(18.0 \mathrm{mg}, \mathrm{t}_{\mathrm{R}}=47.2 \mathrm{~min}\right)$. Fr.3.4.1.3.3.3.3.3 (0.4 g) subjected to chiral Daicel Chiralpak IC column chromatography (n-hexane/ethanol containing $0.05 \%$ TFA, 90:10) to produce 9 (8.82 $\mathrm{mg}$, $\left.\mathrm{t}_{\mathrm{R}}=32.5 \mathrm{~min}\right)$ and $10\left(6.15 \mathrm{mg}, \mathrm{t}_{\mathrm{R}}=24.0 \mathrm{~min}\right)$.

Fr.3.4.1.3.3.4 (1.0 g) was filtered through Sephadex LH-20 $(\mathrm{MeOH})$ to produce four fractions (Fr.3.4.1.3.3.4.1-Fr.3.4.1.3.3.4.4). Fr.3.4.1.3.3.4.4 (110 mg) was subjected to semi-preparative HPLC ( $\mathrm{MeOH}: \mathrm{H}_{2} \mathrm{O}$ containing $0.05 \%$ TFA, $60 \%$, flow rate: $3 \mathrm{~mL} / \mathrm{min}$ ) to form four fractions, the fourth fraction of which was subjected to preparative HPLC (acetonitrile: $\mathrm{MeOH}: \mathrm{H}_{2} \mathrm{O}$ containing 0.05\% TFA, 28:28:44, flow rate: $12 \mathrm{~mL} / \mathrm{min})$ to yield $6\left(37.4 \mathrm{mg}, \mathrm{t}_{\mathrm{R}}=24.2 \mathrm{~min}\right)$.

Fr.3.4.1.1 (39.4 g) was gel filtered though Sephadex LH-20 $(\mathrm{MeOH})$ and then subjected to $\mathrm{C}-18$ silica gel column chromatography $\left(\mathrm{MeOH} / \mathrm{H}_{2} \mathrm{O}, 20-100 \%\right)$ to produce eight fractions (Fr.3.4.1.1.1-Fr.3.4.1.1.8). Fr.3.4.1.1.5 (10 g) was separated into seven parts (Fr.3.4.1.1.5.1-Fr.3.4.1.1.5.7) by using silica gel column chromatography using an eluant comprised of increasing amounts of methanol in dichloromethane. Fr.3.4.1.1.5.5 (2.0 g) was gel filtered through Sephadex $\mathrm{LH}-20(\mathrm{MeOH})$ to obtain five fractions (Fr.3.4.1.1.5.5.1-Fr.3.4.1.1.5.5.5). Fr.3.4.1.1.5.5.5 (0.6 g) was subjected to semi-preparative HPLC $\left(\mathrm{MeOH}: \mathrm{H}_{2} \mathrm{O}\right.$ containing $0.05 \% \mathrm{TFA}, 55 \%$, flow rate: $3 \mathrm{~mL} / \mathrm{min}$ ) to produce four subfractions (Fr.3.4.1.1.5.5.5.1-Fr.3.4.1.1.5.5.5.4). Fr.3.4.1.1.5.5.5.2 (116 mg) was subjected to semi-preparative HPLC (acetonitrile: $\mathrm{MeOH}: \mathrm{H}_{2} \mathrm{O}$ containing 0.05\% TFA, 19:19:62, flow rate: $3 \mathrm{~mL} /$ $\mathrm{min})$ to yield $3\left(10.6 \mathrm{mg}, \mathrm{t}_{\mathrm{R}}=24.2 \mathrm{~min}\right)$. Fr.3.4.1.1.5.5.4 (0.3 g) was divided into two subfractions by using semi-preparative HPLC (MeOH: $\mathrm{H}_{2} \mathrm{O}$ containing 0.05\% TFA, 55\%, flow rate: $3 \mathrm{~mL} /$ $\mathrm{min}$ ), the first subfraction was subjected to chiral Daicel Chiralpak IC column chromatography (n-hexane/ethanol containing 0.05\% TFA, 90:10) to yield $4\left(18.2 \mathrm{mg}, \mathrm{t}_{\mathrm{R}}=19.7 \mathrm{~min}\right)$ and $5\left(17.6 \mathrm{mg}, \mathrm{t}_{\mathrm{R}}=24\right.$. $1 \mathrm{~min})$.

Meroterpenoid $6(2.5 \mathrm{mg})$ was also isolated from $40 \mathrm{~kg}$ Ganoderma lucidum cultivated in Yongsheng County of Yunnan Province, China. This material was also authenticated by Prof. Zhu-Liang Yang at Kunming Institute of Botany, Chinese Academy of Sciences, China. A voucher specimen (CHYX-0609) is deposited at Institute for Inheritance-Based Innovation of Chinese Medicine, Health Science Center, Shenzhen University, China.

\section{Compound Characterization}

Ganodercin $G(1)$, yellow gum; $[\alpha]_{\mathrm{D}}{ }^{20}-14.5$ (c 0.06, MeOH); UV $(\mathrm{MeOH}) \lambda_{\max }(\log \varepsilon) 362$ (3.40), 201 (4.29) nm; CD (MeOH) $\Delta \varepsilon_{202}-1.83$, HRESIMS $m / z$ 375.1812 $[\mathrm{M}+\mathrm{H}]^{+}$, (calcd for $\left.\mathrm{C}_{21} \mathrm{H}_{27} \mathrm{O}_{6}, 375.1802\right),{ }^{1} \mathrm{H}$ and ${ }^{13} \mathrm{C}$ NMR data, see Tables 1 and 2.

Ganodercin $H$ (3), yellow gum; $[\alpha]_{\mathrm{D}}{ }^{20}+10.6$ (c 0.09, MeOH); $\mathrm{CD}(\mathrm{MeOH}) \Delta \varepsilon_{361}-1.52, \Delta \varepsilon_{211}+1.18 ; \mathrm{UV}(\mathrm{MeOH}) \lambda_{\max }(\log \varepsilon)$
361 (3.52), 261 (3.74), 216 (4.26) nm, HRESIMS $m / z 375.1804$ $[\mathrm{M}+\mathrm{H}]^{+}$, (calcd for $\left.\mathrm{C}_{21} \mathrm{H}_{27} \mathrm{O}_{6}, 375.1802\right),{ }^{1} \mathrm{H}$ and ${ }^{13} \mathrm{C}$ NMR data, see Tables 1 and 2.

Ganodercin I (4), yellow gum; $[\alpha]_{\mathrm{D}}^{20}+37.6$ (c 0.11, MeOH); UV (MeOH) $\lambda_{\max }(\log \varepsilon) 297$ (3.66), 223 (4.19), 202 (4.33) nm, $\mathrm{CD}(\mathrm{MeOH}) \Delta \varepsilon_{299}+0.83, \Delta \varepsilon_{260}-0.31, \Delta \varepsilon_{216}+4.43$, HRESIMS $m / z$ $361.2012[\mathrm{M}+\mathrm{H}]^{+}$, (calcd for $\left.\mathrm{C}_{21} \mathrm{H}_{29} \mathrm{O}_{5}, 361.2010\right),{ }^{1} \mathrm{H}$ and ${ }^{13} \mathrm{C}$ NMR data, Tables 1 and 2.

Ganodercin J (5), yellow gum; $[\alpha]_{\mathrm{D}}{ }^{20}-5.9$ (c 0.10, MeOH); UV $(\mathrm{MeOH}) \lambda_{\max }(\log \varepsilon) 296$ (3.54), 222 (3.83), 201 (4.31) nm, CD $(\mathrm{MeOH}) \Delta \varepsilon_{204}+2.89$, HRESIMS $m / z 361.2008[\mathrm{M}+\mathrm{H}]^{+}$, (calcd for $\left.\mathrm{C}_{21} \mathrm{H}_{29} \mathrm{O}_{5}, 361.2010\right),{ }^{1} \mathrm{H}$ and ${ }^{13} \mathrm{C}$ NMR data, see Tables 1 and 2.

Ganodercin $K(7)$, yellow gum; $[\alpha]_{\mathrm{D}}{ }^{20}-35.0$ (c 0.08, MeOH); $\mathrm{CD}(\mathrm{MeOH}) \Delta \varepsilon_{257}-0.40, \Delta \varepsilon_{231}-1.38, \Delta \varepsilon_{215}+1.62, \Delta \varepsilon_{200}-4.86$; UV $(\mathrm{MeOH}) \lambda_{\max }(\log \varepsilon) 364$ (3.55), 256 (3.80), 225 (4.14), 201 (4.21) nm, HRESIMS $m / z 377.1964[\mathrm{M}+\mathrm{H}]^{+}$, (calcd for $\mathrm{C}_{21} \mathrm{H}_{29} \mathrm{O}_{6}$, 377.1959), ${ }^{1} \mathrm{H}$ and ${ }^{13} \mathrm{C}$ NMR data, see Tables 1 and 2.

3-epi-Ganodercin $K(8)$, yellow gum; $[\alpha]_{\mathrm{D}}{ }^{20}-5.0$ (c 0.02 , $\mathrm{MeOH}) ; \mathrm{CD}(\mathrm{MeOH}) \Delta \varepsilon_{259}+0.32, \Delta \varepsilon_{227}+1.38, \Delta \varepsilon_{216}+2.18$, $\Delta \varepsilon_{201}$-3.21; UV (MeOH) $\lambda_{\max }(\log \varepsilon) 364$ (3.61), 256 (3.87), 225 (4.21), 201 (4.30) nm, HRESIMS $m / z 377.1964[\mathrm{M}+\mathrm{H}]^{+}$, (calcd for $\left.\mathrm{C}_{21} \mathrm{H}_{29} \mathrm{O}_{6}, 377.1959\right),{ }^{1} \mathrm{H}$ and ${ }^{13} \mathrm{C} \mathrm{NMR}$ data, see Tables 1 and 2.

Ganodercin $L$ (9), yellow gum; $[\alpha]_{\mathrm{D}}{ }^{20}+12.0($ c $0.03, \mathrm{MeOH})$; $\mathrm{CD}(\mathrm{MeOH}) \Delta \varepsilon_{255}+0.38, \Delta \varepsilon_{228}+1.48, \Delta \varepsilon_{205}+0.75, \Delta \varepsilon_{196}-1.02$; UV (MeOH) $\lambda_{\max }(\log \varepsilon) 363$ (3.48), 256 (3.72), 226 (4.00), 202 (4.01) nm, HRESIMS $\mathrm{m} / z 377.1961[\mathrm{M}+\mathrm{H}]^{+}$, (calcd for $\left.\mathrm{C}_{21} \mathrm{H}_{29} \mathrm{O}_{6}, 377.1959\right),{ }^{1} \mathrm{H}$ and ${ }^{13} \mathrm{C}$ NMR data, see Tables 2 and 3. 3-epi-Ganodercin $L$ (10), yellow gum; $[\alpha]_{\mathrm{D}}{ }^{20}-17.3$ (c 0.08 , $\mathrm{MeOH}) ; \mathrm{CD}(\mathrm{MeOH}) \Delta \varepsilon_{256}-0.13, \Delta \varepsilon_{228}-0.49, \Delta \varepsilon_{201}+0.31$, $\Delta \varepsilon_{192}-1.74$; UV (MeOH) $\lambda_{\max }(\log \varepsilon) 363$ (3.35), 256 (3.59), 227 (3.88), 201 (3.89) nm, HRESIMS $m / z 377.1964[\mathrm{M}+\mathrm{H}]^{+}$, (calcd for $\mathrm{C}_{21} \mathrm{H}_{29} \mathrm{O}_{6}, 377.1959$ ), ${ }^{1} \mathrm{H}$ and ${ }^{13} \mathrm{C}$ NMR data, see Tables 2 and 3.

Ganodercin $M$ (11), yellow gum; $[\alpha]_{\mathrm{D}}{ }^{20}-16.7$ (c 0.03, MeOH); $\mathrm{CD}(\mathrm{MeOH}) \Delta \varepsilon_{256}-0.18, \Delta \varepsilon_{230}-0.70, \Delta \varepsilon_{202}+0.66, \Delta \varepsilon_{192}-1.75$; UV $(\mathrm{MeOH}) \lambda_{\max }(\log \varepsilon) 363$ (3.28), 257 (3.58), 227 (3.88), 201 (3.89) $\mathrm{nm}$, HRESIMS $m / z 391.2120[\mathrm{M}+\mathrm{H}]^{+}$, (calcd for $\mathrm{C}_{22} \mathrm{H}_{31} \mathrm{O}_{6}$, 391.2115), ${ }^{1} \mathrm{H}$ and ${ }^{13} \mathrm{C}$ NMR data, see Tables 2 and 3.

Ganodercin $N$ (12), yellow gum; $[a]_{\mathrm{D}}{ }^{20}+22.5$ (c 0.04, $\mathrm{MeOH}) ; \mathrm{UV}(\mathrm{MeOH}) \lambda_{\max }(\log \varepsilon) 386$ (3.38), 267 (3.91), 227 (3.89), 200 (4.09) nm, HRESIMS $m / z 375.1812[\mathrm{M}+\mathrm{H}]^{+}$, (calcd for $\left.\mathrm{C}_{21} \mathrm{H}_{27} \mathrm{O}_{6}, 375.1802\right),{ }^{1} \mathrm{H}$ and ${ }^{13} \mathrm{C}$ NMR data, see Tables 2 and 3.

Ganodercin $O$ (13), yellow gum; $[\alpha]_{\mathrm{D}}{ }^{20}+10.0$ (c 0.06, MeOH); $\mathrm{CD}(\mathrm{MeOH}) \Delta \varepsilon_{228}-1.19, \Delta \varepsilon_{216}-2.12, \Delta \varepsilon_{202}+1.65 ; \mathrm{UV}(\mathrm{MeOH})$ $\lambda_{\text {max }}(\log \varepsilon) 364$ (3.49), 219 (4.34) nm, HRESIMS $m / z 375.1805$ $[\mathrm{M}+\mathrm{H}]^{+}$, (calcd for $\left.\mathrm{C}_{21} \mathrm{H}_{27} \mathrm{O}_{6}, 375.1802\right),{ }^{1} \mathrm{H}$ and ${ }^{13} \mathrm{C}$ NMR data, see Tables 2 and 3.

Ganodercin $P(14)$, yellow gum; $[\alpha]_{\mathrm{D}}{ }^{20}+24.0($ c $0.03, \mathrm{MeOH})$; $\mathrm{CD}(\mathrm{MeOH}) \Delta \varepsilon_{254}+0.28, \Delta \varepsilon_{229}+0.83$; UV $(\mathrm{MeOH}) \lambda_{\max }(\log \varepsilon)$ 363 (3.45), 256 (3.71), 226 (4.00) nm, HRESIMS m/z 377.1965 $[\mathrm{M}+\mathrm{H}]^{+}$, (calcd for $\left.\mathrm{C}_{21} \mathrm{H}_{29} \mathrm{O}_{6}, 377.1959\right),{ }^{1} \mathrm{H}$ and ${ }^{13} \mathrm{C}$ NMR data, see Tables 2 and 3.

3-epi-Ganodercin $P$ (15), yellow gum; $[\alpha]_{\mathrm{D}}{ }^{20}-4.0$ (c 0.05 , $\mathrm{MeOH}) ; \mathrm{CD}(\mathrm{MeOH}) \Delta \varepsilon_{256}-0.33, \Delta \varepsilon_{226}-1.24 ; \mathrm{UV}(\mathrm{MeOH})$ $\lambda_{\max }(\log \varepsilon) 363$ (3.38), 256 (3.63), 227 (3.92) nm, HRESIMS 
TABLE 1| ${ }^{1} \mathrm{H}$ NMR (500 MHz) data of $\mathbf{1}, \mathbf{3 - 5}, \mathbf{7}$, and $\mathbf{8}(\delta$ in ppm, $J$ in $\mathrm{Hz})$.

\begin{tabular}{|c|c|c|c|c|c|c|}
\hline & 1 & 3 & 4 & 5 & 7 & 8 \\
\hline No & $\delta_{\mathrm{H}^{a}}$ & $\delta_{\mathrm{H}}^{a}$ & $\delta_{\mathrm{H}}^{a}$ & $\delta_{\mathrm{H}}^{a}$ & $\delta_{\mathrm{H}^{b}}^{b}$ & $\delta_{\mathrm{H}^{b}}^{b}$ \\
\hline $3^{\prime}$ & $7.37 \mathrm{~d}(2.2)$ & $7.36 \mathrm{~d}(2.9)$ & $6.58 d(3.0)$ & $6.58 \mathrm{~d}(3.0)$ & $7.42 \mathrm{~d}(3.0)$ & $7.42 \mathrm{~d}(3.0)$ \\
\hline $5^{\prime}$ & $7.02 \mathrm{dd}(8.9,2.2)$ & 7.02 overlap & $6.50 \mathrm{dd}(8.6,3.0)$ & 6.49 dd $(8.6,3.0)$ & $7.10 \mathrm{dd}(8.9,3.0)$ & $7.10 \mathrm{dd}(8.9,3.0)$ \\
\hline $6^{\prime}$ & $6.81 \mathrm{~d}(8.9)$ & $6.81 \mathrm{~d}(8.9)$ & $6.61 \mathrm{~d}(8.6)$ & $6.60 \mathrm{~d}(8.6)$ & $6.81 \mathrm{~d}(8.9)$ & $6.81 \mathrm{~d}(8.9)$ \\
\hline 1 & - & - & $\begin{array}{l}\text { Ha: } 3.73 \text { dd }(15.0,8.3) \\
\text { Hb: } 3.54 \text { dd }(15.0,7.3)\end{array}$ & $\begin{array}{l}\mathrm{Ha}: 3.67 \mathrm{dd}(15.6,7.8) \\
\mathrm{Hb}: 3.65 \mathrm{dd}(15.6,8.3)\end{array}$ & - & - \\
\hline 2 & $4.12 \mathrm{~s}$ & $\begin{array}{l}\text { Ha: } 4.09 \text { d (18.2); } \\
\text { Hb: } 4.05 \text { d (18.2) }\end{array}$ & 5.89 t-like (7.8) & 6.02 t-like (7.7) & $\begin{array}{l}\text { Ha: } 3.56 \text { dd }(17.9,9.3) \\
\text { Hb: } 3.24 \text { dd }(17.9,4.6)\end{array}$ & $\begin{array}{l}\text { Ha: } 3.57 \text { dd }(17.9,9.3) \\
H b: 3.23 \text { dd }(17.9,4.5)\end{array}$ \\
\hline 3 & - & - & - & - & $3.05 \mathrm{~m}$ & $3.06 \mathrm{~m}$ \\
\hline 4 & $6.90 \mathrm{t}(6.4)$ & 7.02 overlap & Ha: 2.47 m; Hb: 2.03 m & Ha: 2.52 m; Hb: 2.23 m & Ha: 1.82 m; Hb: 1.78 m & Ha: 1.82 m; Hb: 1.74 m \\
\hline 5 & $\begin{array}{l}\text { Ha: } 2.97 \text { dd }(17.7,6.4) \\
\text { Hb: } 2.91 \text { dd }(17.7,6.4)\end{array}$ & $2.45 \mathrm{~m}$ & Ha: 1.71 m; Hb: 1.63 m & Ha: 1.80 m; Hb: 1.39 m & $\begin{array}{l}\text { Ha: } 2.22 \text { td }(13.0,5.6) \\
\text { Hb: } 2.12 \text { td }(13.0,4.6)\end{array}$ & $2.17 \mathrm{~m}$ \\
\hline 6 & - & 1.94 dd $(9.8,4.3)$ & 1.66 brs & 1.66 brs & - & - \\
\hline 8 & $2.07 \mathrm{~m}$ & $\begin{array}{l}\text { Ha: } 2.35 \mathrm{dt}(12.4,4.7) \\
\mathrm{Hb}: 2.07 \mathrm{td}(12.4,4.7)\end{array}$ & $\begin{array}{l}\text { Ha: } 2.23 \text { dt }(12.5,4.4) ; \\
\text { Hb: } 1.84 \text { td }(12.5,4.4)\end{array}$ & 5.22 brs & $2.01 \mathrm{dd}(12.6,6.2)$ & $2.01 \mathrm{dd}(12.4,5.7)$ \\
\hline $\begin{array}{l}9 \\
10\end{array}$ & $\begin{array}{c}\text { Ha: } 1.75 \text { m; Hb: } 1.30 \text { m } \\
3.45 \text { dd }(10.0,2.9)\end{array}$ & $\begin{array}{c}\text { Ha: } 1.81 \mathrm{~m} ; \mathrm{Hb}: 1.52 \mathrm{~m} \\
3.38 \mathrm{dd}(9.9,4.2)\end{array}$ & $\begin{array}{c}\text { Ha: } 1.75 \text { m; Hb: } 1.44 \text { m } \\
3.29 \text { dd }(10.4,4.3)\end{array}$ & $\begin{array}{c}\text { Ha: } 2.11 \mathrm{~m} ; \mathrm{Hb}: 1.94 \mathrm{~m} \\
3.36 \mathrm{dd}(8.9,5.7)\end{array}$ & $\begin{array}{c}\text { Ha: } 1.73 \text { m; Hb: } 1.66 \text { m } \\
3.41 \text { dd }(10.3,3.4)\end{array}$ & $\begin{array}{c}\text { Ha: } 1.73 \mathrm{~m} \text {; Hb: } 1.67 \mathrm{~m} \\
3.41 \mathrm{dd}(10.3,3.4)\end{array}$ \\
\hline 12 & $1.04 \mathrm{~s}$ & $1.01 \mathrm{~s}$ & $0.97 \mathrm{~s}$ & $0.94 \mathrm{~s}$ & $1.09 \mathrm{~s}$ & $1.09 \mathrm{~s}$ \\
\hline 13 & $0.96 \mathrm{~s}$ & $0.72 \mathrm{~s}$ & $0.64 \mathrm{~s}$ & $0.76 \mathrm{~s}$ & $0.98 \mathrm{~s}$ & $0.98 \mathrm{~s}$ \\
\hline 14 & $1.58 \mathrm{~s}$ & Ha: $4.90 \mathrm{~s} ; \mathrm{Hb}: 4.59 \mathrm{~s}$ & $\mathrm{Ha}: 4.84 \mathrm{~s} ; \mathrm{Hb}: 4.61 \mathrm{~s}$ & $1.70 \mathrm{~s}$ & $1.61 \mathrm{~s}$ & $1.61 \mathrm{~s}$ \\
\hline $\mathrm{OH}-1^{\prime}$ & - & - & - & - & $11.59 \mathrm{~s}$ & $11.59 \mathrm{~s}$ \\
\hline
\end{tabular}

${ }^{a}$ Record in methanol- $d_{4}$.

${ }^{b}$ Record in acetone- $d_{6}$.

TABLE $2 \mid{ }^{13} \mathrm{C}$ NMR data of $\mathbf{1}, \mathbf{3 - 5}, \mathbf{7 - 1 5}(\delta$ in ppm).

\begin{tabular}{|c|c|c|c|c|c|c|c|c|c|c|c|c|c|}
\hline & 1 & 3 & 4 & 5 & 7 & 8 & 9 & 10 & 11 & 12 & 13 & 14 & 15 \\
\hline No & $\delta_{\mathrm{C}}^{a}$ & $\delta_{\mathrm{C}}^{a}$ & $\delta_{\mathrm{C}}^{a}$ & $\delta_{\mathrm{C}}^{a}$ & $\delta_{\mathrm{C}}^{b}$ & $\delta_{\mathrm{C}}^{b}$ & $\delta_{C}^{b}$ & $\delta_{\mathrm{C}}^{b}$ & $\delta_{\mathrm{C}}^{a}$ & $\delta_{\mathrm{C}}^{c}$ & $\delta_{\mathrm{C}}^{a}$ & $\delta_{\mathrm{C}}^{a}$ & $\delta_{\mathrm{C}}^{a}$ \\
\hline $1^{\prime}$ & $150.7 \mathrm{~s}$ & $150.7 \mathrm{~s}$ & $149.3 \mathrm{~s}$ & $149.2 \mathrm{~s}$ & $149.3 \mathrm{~s}$ & $149.3 \mathrm{~s}$ & $149.3 \mathrm{~s}$ & $149.3 \mathrm{~s}$ & $150.7 \mathrm{~s}$ & $150.9 \mathrm{~s}$ & $150.5 \mathrm{~s}$ & $150.7 \mathrm{~s}$ & $150.7 \mathrm{~s}$ \\
\hline $2^{\prime}$ & $120.6 \mathrm{~s}$ & $120.6 \mathrm{~s}$ & $127.9 \mathrm{~s}$ & $128.2 \mathrm{~s}$ & $119.1 \mathrm{~s}$ & $119.1 \mathrm{~s}$ & $119.1 \mathrm{~s}$ & $119.1 \mathrm{~s}$ & $120.4 \mathrm{~s}$ & $121.3 \mathrm{~s}$ & $120.4 \mathrm{~s}$ & $120.4 \mathrm{~s}$ & $120.5 \mathrm{~s}$ \\
\hline $3^{\prime}$ & $115.7 \mathrm{~d}$ & $115.6 \mathrm{~d}$ & $117.8 \mathrm{~d}$ & $117.7 \mathrm{~d}$ & $114.6 \mathrm{~d}$ & $114.6 \mathrm{~d}$ & $114.6 \mathrm{~d}$ & $114.6 \mathrm{~d}$ & $115.4 d$ & $115.9 d$ & $115.6 \mathrm{~d}$ & $115.4 d$ & $115.4 d$ \\
\hline $4^{\prime}$ & $156.5 \mathrm{~s}$ & $156.5 \mathrm{~s}$ & $151.2 \mathrm{~s}$ & $151.2 \mathrm{~s}$ & $155.3 \mathrm{~s}$ & $155.3 \mathrm{~s}$ & $155.3 \mathrm{~s}$ & $155.3 \mathrm{~s}$ & $156.5 \mathrm{~s}$ & $157.2 \mathrm{~s}$ & $156.4 \mathrm{~s}$ & $156.5 \mathrm{~s}$ & $156.5 \mathrm{~s}$ \\
\hline $5^{\prime}$ & $125.8 d$ & $125.9 \mathrm{~d}$ & $114.8 d$ & $114.8 \mathrm{~d}$ & $124.7 \mathrm{~d}$ & $124.8 \mathrm{~d}$ & $124.7 \mathrm{~d}$ & $124.7 \mathrm{~d}$ & $126.0 \mathrm{~d}$ & $126.8 d$ & $125.8 d$ & $125.9 d$ & $125.9 \mathrm{~d}$ \\
\hline $6^{\prime}$ & $119.7 \mathrm{~d}$ & $119.7 \mathrm{~d}$ & $116.9 \mathrm{~d}$ & $117.9 \mathrm{~d}$ & $118.5 \mathrm{~d}$ & $118.5 \mathrm{~d}$ & $118.5 d$ & $118.5 \mathrm{~d}$ & $119.7 \mathrm{~d}$ & $120.0 d$ & $119.6 \mathrm{~d}$ & $119.7 \mathrm{~d}$ & $119.7 \mathrm{~d}$ \\
\hline 1 & $204.1 \mathrm{~s}$ & $204.3 \mathrm{~s}$ & $31.7 \mathrm{t}$ & $31.7 \mathrm{t}$ & $204.8 \mathrm{~s}$ & $204.8 \mathrm{~s}$ & $204.8 \mathrm{~s}$ & $204.9 \mathrm{~s}$ & $205.5 \mathrm{~s}$ & $199.2 \mathrm{~s}$ & $203.9 \mathrm{~s}$ & $205.6 \mathrm{~s}$ & $205.6 \mathrm{~s}$ \\
\hline 2 & $37.6 \mathrm{t}$ & $37.7 \mathrm{t}$ & $140.5 \mathrm{~d}$ & $141.1 \mathrm{~d}$ & $39.6 \mathrm{t}$ & $39.6 \mathrm{t}$ & $40.2 \mathrm{t}$ & $39.6 \mathrm{t}$ & $41.8 \mathrm{t}$ & $132.8 d$ & $37.7 \mathrm{t}$ & $41.5 \mathrm{t}$ & $41.2 \mathrm{t}$ \\
\hline 3 & $126.6 \mathrm{~s}$ & $127.0 \mathrm{~s}$ & $133.6 \mathrm{~s}$ & $133.9 \mathrm{~s}$ & $40.3 d$ & $40.2 d$ & $40.0 \mathrm{~d}$ & 39.9 d & $41.7 \mathrm{~d}$ & $146.3 \mathrm{~s}$ & $127.4 \mathrm{~s}$ & $41.9 d$ & $41.8 d$ \\
\hline 4 & $148.6 \mathrm{~d}$ & $148.5 \mathrm{~d}$ & $34.6 \mathrm{t}$ & $37.9 \mathrm{t}$ & $32.1 \mathrm{t}$ & $32.1 \mathrm{t}$ & $31.2 \mathrm{t}$ & $31.1 \mathrm{t}$ & $32.5 \mathrm{t}$ & $29.8 \mathrm{t}$ & $147.7 \mathrm{~d}$ & $33.3 \mathrm{t}$ & $33.1 \mathrm{t}$ \\
\hline 5 & $29.4 t$ & $26.9 \mathrm{t}$ & $25.4 \mathrm{t}$ & $29.3 t$ & $26.2 \mathrm{t}$ & $26.2 \mathrm{t}$ & $23.1 \mathrm{t}$ & $23.0 \mathrm{t}$ & $24.1 \mathrm{t}$ & $28.3 t$ & $28.6 t$ & $26.4 \mathrm{t}$ & $26.2 \mathrm{t}$ \\
\hline 6 & $135.0 \mathrm{~s}$ & $52.8 \mathrm{~d}$ & $51.4 d$ & $50.2 d$ & $135.8 \mathrm{~s}$ & $135.8 \mathrm{~s}$ & $52.1 \mathrm{~d}$ & $52.2 \mathrm{~d}$ & $52.9 \mathrm{~d}$ & $57.1 d$ & $56.8 d$ & $56.8 d$ & $56.8 \mathrm{~d}$ \\
\hline 7 & $129.9 \mathrm{~s}$ & $148.7 \mathrm{~s}$ & $148.8 \mathrm{~s}$ & $137.8 \mathrm{~s}$ & $126.3 \mathrm{~s}$ & $126.3 \mathrm{~s}$ & $148.2 \mathrm{~s}$ & $148.1 \mathrm{~s}$ & $149.0 \mathrm{~s}$ & $88.6 \mathrm{~s}$ & $88.3 \mathrm{~s}$ & $88.6 \mathrm{~s}$ & $88.6 \mathrm{~s}$ \\
\hline 8 & $31.6 \mathrm{t}$ & $33.9 t$ & $34.6 \mathrm{t}$ & $120.1 d$ & $30.4 \mathrm{t}$ & $30.4 \mathrm{t}$ & $32.5 \mathrm{t}$ & $32.5 \mathrm{t}$ & $34.1 \mathrm{t}$ & $39.7 \mathrm{t}$ & $39.2 \mathrm{t}$ & $39.8 t$ & $39.8 \mathrm{t}$ \\
\hline 9 & $27.8 \mathrm{t}$ & $32.8 \mathrm{t}$ & $33.1 \mathrm{t}$ & $32.5 t$ & $27.1 \mathrm{t}$ & $27.1 \mathrm{t}$ & $32.2 \mathrm{t}$ & $32.2 \mathrm{t}$ & $33.1 \mathrm{t}$ & $26.5 \mathrm{t}$ & $26.7 \mathrm{t}$ & $26.6 \mathrm{t}$ & $26.6 \mathrm{t}$ \\
\hline 10 & $76.6 \mathrm{~d}$ & $77.5 \mathrm{~d}$ & $77.9 \mathrm{~d}$ & $75.6 \mathrm{~d}$ & $74.8 \mathrm{~d}$ & $74.8 \mathrm{~d}$ & $75.7 \mathrm{~d}$ & $75.6 \mathrm{~d}$ & $77.7 \mathrm{~d}$ & $87.6 \mathrm{~d}$ & $87.5 \mathrm{~d}$ & $87.7 \mathrm{~d}$ & $87.7 \mathrm{~d}$ \\
\hline 11 & $41.3 \mathrm{~s}$ & $41.5 \mathrm{~s}$ & $41.6 \mathrm{~s}$ & $39.3 \mathrm{~s}$ & $40.1 \mathrm{~s}$ & $40.1 \mathrm{~s}$ & $40.4 \mathrm{~s}$ & $40.4 \mathrm{~s}$ & $41.9 \mathrm{~s}$ & $46.4 \mathrm{~s}$ & $46.2 \mathrm{~s}$ & $46.3 \mathrm{~s}$ & $46.4 \mathrm{~s}$ \\
\hline 12 & $26.2 \mathrm{q}$ & $26.3 \mathrm{q}$ & 26.2 q & $25.7 \mathrm{q}$ & $25.4 \mathrm{q}$ & $25.4 \mathrm{q}$ & $25.7 \mathrm{q}$ & $26.4 \mathrm{q}$ & $26.5 \mathrm{q}$ & $18.9 \mathrm{q}$ & $26.1 \mathrm{q}$ & $26.3 \mathrm{q}$ & $26.2 \mathrm{q}$ \\
\hline 13 & $21.5 \mathrm{q}$ & $16.0 \mathrm{q}$ & $15.5 \mathrm{q}$ & $15.8 \mathrm{q}$ & $20.8 q$ & $20.9 q$ & $15.7 \mathrm{q}$ & $15.8 \mathrm{q}$ & $16.1 \mathrm{q}$ & $26.0 \mathrm{q}$ & $24.2 \mathrm{q}$ & $23.7 \mathrm{q}$ & $23.7 \mathrm{q}$ \\
\hline 14 & $19.9 \mathrm{q}$ & $110.0 \mathrm{t}$ & $108.6 t$ & $22.7 \mathrm{q}$ & $18.9 \mathrm{q}$ & $18.9 \mathrm{q}$ & $107.6 \mathrm{t}$ & 107.6 t & $108.7 \mathrm{t}$ & $23.7 \mathrm{q}$ & $19.1 \mathrm{q}$ & $19.0 \mathrm{q}$ & $19.0 \mathrm{q}$ \\
\hline 15 & $170.5 \mathrm{~s}$ & $170.5 \mathrm{~s}$ & $172.3 \mathrm{~s}$ & $172.0 \mathrm{~s}$ & $175.4 \mathrm{~s}$ & $175.5 \mathrm{~s}$ & $175.6 \mathrm{~s}$ & $175.6 \mathrm{~s}$ & $177.7 \mathrm{~s}$ & $170.2 \mathrm{~s}$ & $170.3 \mathrm{~s}$ & $179.0 \mathrm{~s}$ & $178.9 \mathrm{~s}$ \\
\hline $\mathrm{OCH}_{3}$ & - & - & - & - & - & - & - & - & $52.2 \mathrm{q}$ & - & - & - & - \\
\hline
\end{tabular}

${ }^{a}$ Record in methanol- $d_{4}$ at $125 \mathrm{MHz}$

${ }^{b}$ Record in acetone- $d_{6}$ at $125 \mathrm{MHz}$.

${ }^{c}$ Record in methanol- $d_{4}$ at $150 \mathrm{MHz}$.

$m / z 377.1968[\mathrm{M}+\mathrm{H}]^{+}$, (calcd for $\left.\mathrm{C}_{21} \mathrm{H}_{29} \mathrm{O}_{6}, 377.1959\right),{ }^{1} \mathrm{H}$ and ${ }^{13} \mathrm{C}$ NMR data, see Tables 2 and 3.

Crystal Data for $\mathrm{C}_{21} \mathrm{H}_{28} \mathrm{O}_{6}(M=376.43 \mathrm{~g} / \mathrm{mol})$ : monoclinic, space group P2 1 (no. 4), $a=6.62350(10) \AA, b=11.59270(10) \AA$, $c=13.40440(10) \AA, \beta=100.4300(10)^{\circ}, V=1012.240(19) \AA^{3}, Z=$
2, $T=99.9(9) \mathrm{K}, \mu(\mathrm{CuK} \alpha)=0.737 \mathrm{~mm}^{-1}$, Dcalc $=1.235 \mathrm{~g} / \mathrm{cm}^{3}$, 9814 reflections measured $\left(6.704^{\circ} \leq 2 \Theta \leq 148.606^{\circ}\right), 3973$ unique $\left(R_{\text {int }}=0.0305, R_{\text {sigma }}=0.0308\right)$ which were used in all calculations. The final $R_{1}$ was $0.0324(\mathrm{I}>2 \sigma(\mathrm{I}))$ and $w R_{2}$ was 0.0865 (all data). Crystallographic data of 3-epi-ganodercin $\mathrm{P}$ 
TABLE 3 $\mid{ }^{1} \mathrm{H}$ NMR data of $\mathbf{9 - 1 5}$ in methanol- $d_{4}(\delta$ in ppm, $J$ in $\mathrm{Hz}$ ).

\begin{tabular}{|c|c|c|c|c|c|c|c|}
\hline & 9 & 10 & 11 & 12 & 13 & 14 & 15 \\
\hline No & $\delta_{\mathrm{H}}^{a}$ & $\delta_{\mathrm{H}}^{a}$ & $\delta_{\mathrm{H}}^{b}$ & $\delta_{\mathrm{H}}^{c}$ & $\delta_{\mathrm{H}}^{b}$ & $\delta_{\mathrm{H}}^{b}$ & $\delta_{\mathrm{H}}^{b}$ \\
\hline $3^{\prime}$ & $7.40 \mathrm{~d}(2.9)$ & $7.42 \mathrm{~d}(2.9)$ & $7.23 \mathrm{~d}(2.9)$ & $7.15 \mathrm{~d}(3.0)$ & $7.35 \mathrm{~d}(3.0)$ & $7.26 \mathrm{~d}(2.9)$ & $7.26 \mathrm{~d}(2.9)$ \\
\hline $5^{\prime}$ & $7.10 \mathrm{dd}(8.9,2.9)$ & 7.09 dd $(8.9,2.9)$ & $7.00 \mathrm{dd}(8.9,2.9)$ & 7.05 dd $(8.9,3.0)$ & $7.01 \mathrm{dd}(8.9,3.0)$ & $7.01 \mathrm{dd}(8.9,2.9)$ & $7.01 \mathrm{dd}(8.9,3.0)$ \\
\hline $6^{\prime}$ & $6.81 \mathrm{~d}(8.9)$ & $6.81 \mathrm{~d}(8.9)$ & $6.79 \mathrm{~d}(8.9)$ & $6.84 \mathrm{~d}(8.9)$ & $6.80 \mathrm{~d}(8.9)$ & $6.79 \mathrm{~d}(8.9)$ & $6.79 \mathrm{~d}(8.9)$ \\
\hline 2 & $\begin{array}{c}\mathrm{Ha}: 3.51 \mathrm{dd}(17.8 \\
9.3) ; \mathrm{Hb}: 3.17 \mathrm{dd} \\
(17.8,4.5)\end{array}$ & $\begin{array}{c}\text { Ha: } 3.52 \text { dd (17.9, } \\
9.6) ; ~ H b: 3.19 \text { dd } \\
(17.9,4.2)\end{array}$ & $\begin{array}{c}\text { Ha: } 3.42 \text { dd (17.9, } \\
9.5) ; ~ H b: 3.15 \mathrm{dd} \\
(17.9,4.6)\end{array}$ & $7.64 \mathrm{~s}$ & $\begin{array}{l}\mathrm{Ha}: 4.09 \text { d (18.0) } \\
\mathrm{Hb}: 4.04 \mathrm{~d}(18.0)\end{array}$ & $\begin{array}{c}\text { Ha: } 3.44 \text { dd (17.9, } \\
9.1) ; \text { Hb: } 3.14 \text { dd } \\
(17.9,4.8)\end{array}$ & $\begin{array}{c}\text { Ha: } 3.44 \text { dd (17.8, } \\
9.1) ; ~ H b: 3.14 \text { dd } \\
(17.8,4.8)\end{array}$ \\
\hline 3 & $3.02 \mathrm{~m}$ & $3.03 \mathrm{~m}$ & $2.97 \mathrm{~m}$ & - & - & $2.95 \mathrm{~m}$ & $2.97 \mathrm{~m}$ \\
\hline 4 & $\begin{array}{c}\text { Ha: } 1.86 \text { m; Hb: } \\
1.44 \text { m }\end{array}$ & $\begin{array}{c}\text { Ha: } 1.82 \text { m; Hb: } \\
1.54 \text { m }\end{array}$ & $\begin{array}{c}\text { Ha: } 1.82 \text { m; Hb: } \\
1.38 \text { m }\end{array}$ & $\begin{array}{c}\mathrm{Ha:} 2.58 \mathrm{~m} ; \mathrm{Hb}: \\
2.52 \mathrm{~m}\end{array}$ & 7.07 t (6.8) & $\begin{array}{c}\text { Ha: } 1.68 \text { m; Hb: } \\
1.59 \text { m }\end{array}$ & $\begin{aligned} \text { Ha: } & 1.69 \text { m; Hb: } \\
& 1.63 \text { m }\end{aligned}$ \\
\hline 5 & $\begin{array}{c}\text { Ha: } 1.73 \mathrm{~m} ; \mathrm{Hb}: \\
1.69 \mathrm{~m}\end{array}$ & $\begin{array}{c}\text { Ha: } 1.74 \text { m; Hb: } \\
1.71 \mathrm{~m}\end{array}$ & Ha: 1.60 m & $\begin{array}{c}\mathrm{Ha:} 1.51 \mathrm{~m} ; \mathrm{Hb}: \\
1.46 \mathrm{~m}\end{array}$ & $2.20 \mathrm{~m}$ & $1.47 \mathrm{~m}$ & $\begin{array}{c}\text { Ha: } 1.46 \text { m; Hb: } \\
1.36 \text { m }\end{array}$ \\
\hline 6 & 1.74 brs & $1.72 \mathrm{~m}$ & 1.70 dd $(8.3,4.3)$ & $1.26 \mathrm{t}(7.3)$ & $1.58 \mathrm{t}(7.7)$ & $1.29 \mathrm{dd}(5.2,3.1)$ & $1.29 \mathrm{dd}(8.2,5.8)$ \\
\hline 8 & $\begin{array}{c}\text { Ha: } 2.33 \mathrm{dt}(12.9 \\
5.0) ; \mathrm{Hb}: 2.00 \mathrm{dt} \\
(12.9,4.8)\end{array}$ & $\begin{array}{c}\text { Ha: } 2.34 \mathrm{dt}(12.9 \\
5.0) ; \mathrm{Hb}: 2.00 \mathrm{dt} \\
(12.9,4.9)\end{array}$ & $\begin{array}{c}\mathrm{Ha}: 2.32 \mathrm{dt}(12.9 \\
\text { 4.8); } \mathrm{Hb}: 2.00 \mathrm{dt} \\
(12.9,4.3)\end{array}$ & $\begin{array}{l}\text { Ha: } 1.51 \text { m; Hb: } \\
1.39 \text { td }(12.1,4.5)\end{array}$ & $\begin{array}{c}\text { Ha: } 1.62 \text { m; Hb: } \\
1.45 \text { m }\end{array}$ & $\begin{array}{c}\text { Ha: } 1.59 \text { m; Hb: } \\
1.46 \text { m }\end{array}$ & $\begin{array}{c}\text { Ha: } 1.59 \text { m; Hb: } \\
1.46 \text { m }\end{array}$ \\
\hline 9 & $\begin{array}{c}\text { Ha: } 1.80 \text { m; Hb: } \\
1.52 \text { m }\end{array}$ & $\begin{array}{c}\text { Ha: } 1.80 \text { m; Hb: } \\
1.50 \text { m }\end{array}$ & $\begin{array}{c}\text { Ha: } 1.80 \text { m; Hb: } \\
1.52 \text { m }\end{array}$ & $\begin{array}{c}\text { Ha: } 1.94 \text { m; Hb: } \\
1.63 \text { m }\end{array}$ & $\begin{array}{c}\text { Ha: } 1.99 \text { m; Hb: } \\
1.67 \text { m }\end{array}$ & $\begin{array}{c}\text { Ha: } 1.99 \text { m; Hb: } \\
1.67 \text { m }\end{array}$ & $\begin{array}{c}\text { Ha: } 1.98 \text { m; Hb: } \\
1.66 \text { m }\end{array}$ \\
\hline 10 & 3.39 dd $(9.4,4.1)$ & 3.39 dd $(9.3,4.1)$ & $3.36 \mathrm{dd}(9.9,4.2)$ & 3.70 brd (5.4) & $\begin{array}{c}3.41 \mathrm{dd} \\
(10.3,3.4)\end{array}$ & 3.73 brd (5.3) & 3.73 brd (5.3) \\
\hline 12 & $1.04 \mathrm{~s}$ & $1.04 \mathrm{~s}$ & $1.03 \mathrm{~s}$ & $1.02 \mathrm{~s}$ & $1.08 \mathrm{~s}$ & $1.09 \mathrm{~s}$ & $1.09 \mathrm{~s}$ \\
\hline 13 & $0.75 \mathrm{~s}$ & $0.75 \mathrm{~s}$ & $0.71 \mathrm{~s}$ & $0.97 \mathrm{~s}$ & $0.97 \mathrm{~s}$ & $1.01 \mathrm{~s}$ & $1.01 \mathrm{~s}$ \\
\hline 14 & Ha: 4.85 s; Hb: 4.64 s & Ha: $4.86 \mathrm{~s} ; \mathrm{Hb}: 4.65 \mathrm{~s}$ & $\begin{array}{c}\text { Ha: } 4.86 \mathrm{~s} ; \mathrm{Hb}: \\
4.55 \mathrm{~s}\end{array}$ & $1.28 \mathrm{~s}$ & $1.30 \mathrm{~s}$ & $1.32 \mathrm{~s}$ & $1.32 \mathrm{~s}$ \\
\hline $\mathrm{OCH}_{3}$ & - & - & $3.68 \mathrm{~s}$ & - & - & - & - \\
\hline $\mathrm{OH}-1^{\prime}$ & $11.59 \mathrm{~s}$ & $11.59 \mathrm{~s}$ & - & - & - & - & - \\
\hline $\mathrm{OH}-4^{\prime}$ & $8.15 \mathrm{~s}$ & $8.15 \mathrm{~s}$ & - & - & - & - & - \\
\hline
\end{tabular}

${ }^{a}$ Record in acetone- $d_{6}$ at $500 \mathrm{MHz}$.

${ }^{b}$ Record in methanol- $d_{4}$ at $500 \mathrm{MHz}$.

${ }^{c}$ Record in methanol- $d_{4}$ at $600 \mathrm{MHz}$.

(15) have been deposited at the Cambridge Crystallographic Data Centre (CCDC deposition no. 2105988).

\section{Renal Fibrosis Activity Assay}

TGF- $\beta 1$ induced rat renal proximal tubular cells (NRK-52E) were used to assess expression of the target gene. The cell culture method, and cell viability and western blotting assays were conducted following our previously reported protocols (Meng et al., 2021).

\section{Biological Activity Assay on Triple Negative Breast Cancer Cell Lines (MDA-MB-231, BT549 and HCC 1806)}

\section{Cell Culture}

MDA-MB-231 (ATCC, Cat No. HTB-26, United States), BT549 (ATCC, Cat No. HTB-122, United States) and HCC1806 (ATCC, Cat No. CRL-2335, United States) cells were cultured in Dulbecco's Modified Eagle Medium (HyClone, Cat No.SH30243.01, United States) or RPMI Medium Modified (HyClone, Cat No.SH30809.01, United States) supplemented with $10 \%$ fetal bovine serum (HyClone, Cat No.SV30160.03, United States) and $1 \%$ penicillin/ streptomycin (Hyclone, Cat No.SV30010, United States) in $\mathrm{CO}_{2}(5 \%)$ incubator at $37^{\circ} \mathrm{C}$.

\section{Cell Viability Assay}

Cell viability was evaluated by using a CCK- 8 assay kit (meilunbio, Cat No. MA0218, China) according to the manufacturer's instructions. MDA-MB-231, BT549 and HCC1806 cells were seeded into 96-well plates with $3 \times 10^{3}$ cells per well. After $72 \mathrm{~h}$ exposure in the medium containing desired compounds $(20 \mu \mathrm{M}), 100 \mu \mathrm{L}$ fresh medium including $10 \mu \mathrm{L}$ CCK- 8 reagent was added, and then the plates were incubated at $37^{\circ} \mathrm{C}$ for $2 \mathrm{~h}$. The absorbances of CCK- 8 in each well was measured at 450 and $600 \mathrm{~nm}$ by using a Cytation 5 (BioTek, United States). DMSO was used as a control. Each sample was plated in triplicate.

\section{Wound Healing Assays}

Confluent MDA-MB-231, BT549, and HCC1806 cells were wounded by scratching using Wound Making Tool-Auto Scratch (BioTek, United States). After exposure for $24 \mathrm{~h}$ in the medium containing desired compounds $(20 \mu \mathrm{M})$, the scratched gap area of each cell monolayer was photographed by using Cytation5 (BioTek, United States) and quantified by using Image-Pro Plus 6.0. (http://rsb. info.nih.gov/ij/download.html). The migration efficiency of each cell was then determined as average percentage of closure of the scratch area. Each sample was plated in triplicate. All product recommended protocols were followed. 
<smiles>[Y]C1=C(C/C=C(/CC(=O)c2cc(O)ccc2O)C(=O)O)[C@]([Y20])(C)[C@H](O)CC1</smiles>

1<smiles>CC1=CC[C@@H](O)C(C)(C)[C@H]1CC/C(=C/Cc1cc(O)ccc1O)C(=O)O</smiles>

5<smiles>C=C1CC[C@@H](O)C(C)(C)[C@H]1CC[C@H](CC(=O)c1cc(O)ccc1O)C(=O)OC</smiles>

11<smiles>CC1=C(CC/C(=C\C(=O)c2cc(O)ccc2O)C(=O)O)[C@](C)(O)CC[C@H]1C</smiles><smiles>CC1=CC[C@@H](O)C(C)(C)[C@H]1C/C=C(\CC(=O)c1cc(O)ccc1O)C(=O)O</smiles>

6<smiles>CC1(C)[C@@H]2CC[C@@]1(C)[C@@H]2CC/C(=C\C(=O)c1cc(O)ccc1O)C(=O)O</smiles>

12<smiles>C=C1CCC(O)C(C)(C)[C@H]1/C=C/C(C(=O)O)=C(CC(=O)c1cc(O)ccc1O)C(=O)O</smiles><smiles>C=C1CC[C@@H](O)C(C)(C)[C@H]1CC/C(=C/Cc1cc(O)ccc1O)C(=O)O</smiles><smiles>CC1=C(CCC(CC(=O)c2cc(O)ccc2O)C(=O)O)C(C)[C@H](O)CC1</smiles><smiles>C=C1CC[C@@H](O)C(C)(C)[C@H]1CC[C@H](CC(=O)c1cc(O)ccc1O)C(=O)O</smiles>

$73 R$
$83 S$

$93 S$

$103 R$<smiles>CC1(C)[C@H]2C=C[C@@H]1C(C)(C)[C@H]2/C=C/C(CC(=O)c1cc(O)ccc1O)C(=O)O</smiles>

13

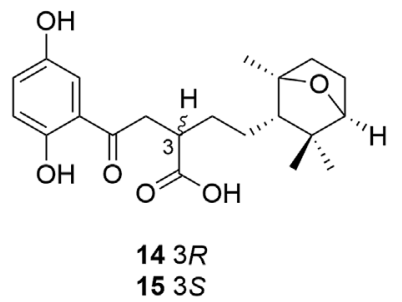

FIGURE 1 | Structures of 1-15 isolated from G. cochlear.

\section{Western Blot Analysis}

The total proteins of the whole cell lysates were separated by using SDS-PAGE and transferred to a PVDF membrane (Millipore, Cat No. IPVH00010, United States). After being probed with primary antibodies overnight at $4^{\circ} \mathrm{C}$, the proteins of interest were then detected using HRP-conjugated IgG (CST, Cat No.7074P2, United States) and visualized by using ECL substrate (4ABiotech, Cat No.4AW011, China) based imaging with a Minichemi ${ }^{\mathrm{TM}}$ chemiluminescence imaging system (SAGECREATION, China).

\section{Statistical Analysis}

Analysis of statistical data, obtained from triplicate measurements, was performed by using the Student's $t$-test for two groups or by one-way ANOVA for multiple groups. $\# p<0.05$ was considered to be significant.

\section{Biological Activity Assay on L6 Myotubes Cells \\ Cell Culture}

L6 myotubes cells were maintained in $\alpha$-MEM culture medium supplemented with $10 \%$ (v/v) fetal bovine serum (FBS), 100 $\mathrm{U} / \mathrm{mL}$ penicillin-streptomycin, and incubated at $37^{\circ} \mathrm{C}$ in an atmosphere of $5 \% \mathrm{CO}_{2}$.

\section{Western Blotting Analysis}

Cells were washed with pre-cold PBS, lysed using RIPA buffer supplemented with Proteinase Inhibitors Cocktail (MCE), and PMSF (MCE). AMPK, p-AMPK, AKT, p-AKT and GAPDH antibody were incubated overnight and the secondary antibody was incubated for $1 \mathrm{~h}$ at room temperature. Signals were detected using a Western blotting Imaging System according to the manufacturer's specifications. The following primary antibodies were used for blotting: p-AKT (4060S, CST), AKT (9272S, CST), p-AMPK (2535S, CST), AMPK (2532, CST) and GAPDH (5174S, CST).

\section{RESULTS AND DISCUSSION}

Powders of the dried fruiting bodies of the fungus G. cochlear $(200 \mathrm{~kg})$ were first extracted with refluxing $80 \% \mathrm{EtOH}$ and partitioned in water to obtain an ethyl acetate soluble fractions, an qliquote was then subjected to multiple chromatographic separation steps. This procedure led to isolation of fifteen the cyclohexane-type meroterpenoids 1-15 (Figure 1).

Ganodercin G (1), obtained as a yellow gum, has a molecular formula of $\mathrm{C}_{21} \mathrm{H}_{26} \mathrm{O}_{6}$ (nine degrees of unsaturation) established by using HRESIMS $(\mathrm{m} / z 375.1812$ $[\mathrm{M}+\mathrm{H}]^{+}$, calcd for 375.1802), and ${ }^{13} \mathrm{C} \mathrm{NMR}$ and DEPT spectroscopy. The presence of a 1,2,4-trisubstituted benzene ring in $\mathbf{1}$ was assigned by analysis of the ${ }^{1} \mathrm{H}$ NMR spectrum of 1 (Table 1), which contain resonances in the aromatic region $\left(\delta_{\mathrm{H}} 7.37, \mathrm{~d}, J=2.2 \mathrm{~Hz}, \mathrm{H}-3^{\prime} ; \delta_{\mathrm{H}} 7.02, \mathrm{dd}, J=8.9,2.2 \mathrm{~Hz}, \mathrm{H}-5^{\prime}\right.$; $\left.\delta_{\mathrm{H}} 6.81, \mathrm{~d}, J=8.9 \mathrm{~Hz}, \mathrm{H}-6^{\prime}\right)$. The ${ }^{13} \mathrm{C}$ NMR and DEPT data of 1 (Table 2) revealed the presence of three methyls, four $\mathrm{sp}^{3}$ methylenes, five methines (four $\mathrm{sp}^{2}$ and one $\mathrm{sp}^{3}$ ) and nine 


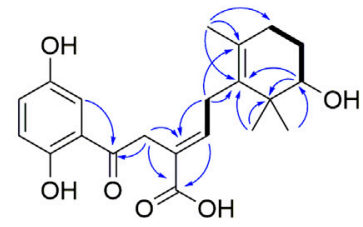

1

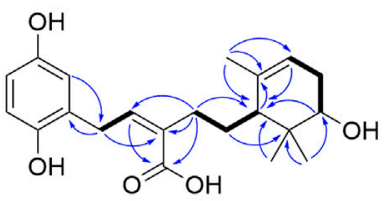

5

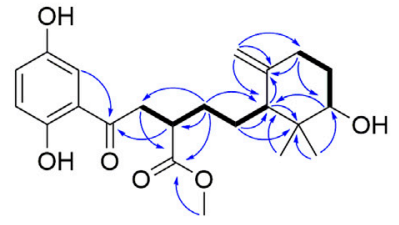

11

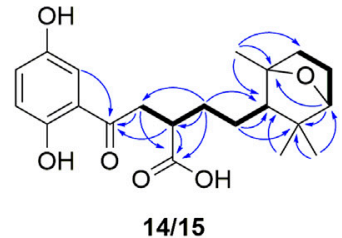

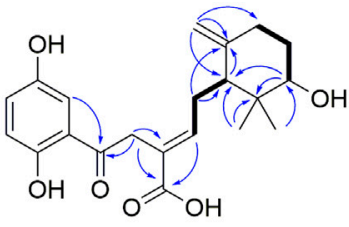

3

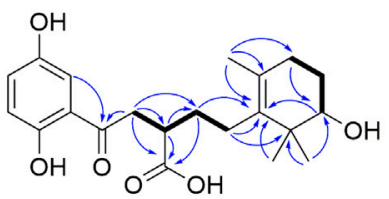

$7 / 8$

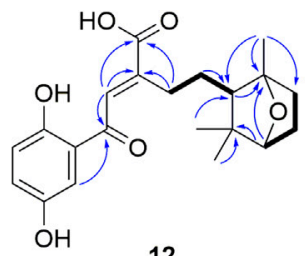

12

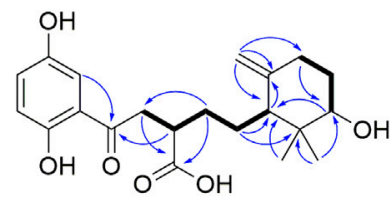

13
$9 / 10$
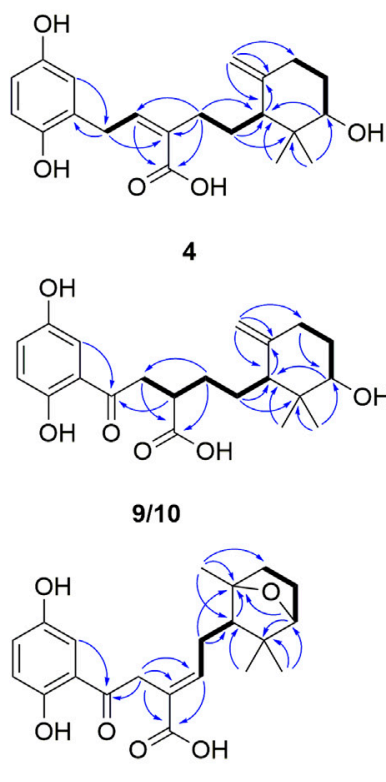

4

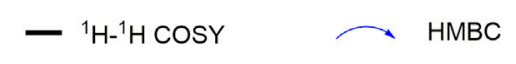

FIGURE 2 | Key COSY and HMBC correlations of 1, 3-5, 7-15.

nonprotonated carbons (one ketone, one carbonyl, six aromatic including two oxygenated and one aliphatic). The NMR spectra of $\mathbf{1}$ are similar to those of (-)-ganotheaecoloid F (Luo et al., 2018), which was isolated from the fruiting bodies of $G$. theaecolum, with the main difference being the presence of a $\Delta^{3(4)}$ double bond in 1 instead of the $\Delta^{2(3)}$ double bond in (-)-ganotheaecoloid $\mathrm{F}$. This difference is supported by the ${ }^{1} \mathrm{H}-{ }^{1} \mathrm{H}$ COSY correlation of $\mathrm{H}-4\left(\delta_{\mathrm{H}} 6.90\right) / \mathrm{H}_{2}-5\left(\delta_{\mathrm{H}} 2.97\right.$ and $2.91)$ and the HMBC (Figure 2) correlations of $\mathrm{H}_{2}-2\left(\delta_{\mathrm{H}}\right.$ $4.12) / \mathrm{C}-3\left(\delta_{\mathrm{C}} 126.6\right), \mathrm{C}-15\left(\delta_{\mathrm{C}} 170.5\right), \mathrm{H}-4 / \mathrm{C}-15$, and $\mathrm{H}_{2}-5 / \mathrm{C}-3$. The ROESY correlation (Figure 3 ) of $\mathrm{H}-2 / \mathrm{H}-5$ suggested that the $\Delta^{3(4)}$ double bond in $\mathbf{1}$ has $E$ configuration. Chiral HPLC analysis revealed that compound $\mathbf{1}$ is enantiomerically pure. Time-dependent density functional theory (TDDFT) ECD calculations were used to determine the absolute configuration at the stereogenic center in $\mathbf{1}$. It was found that the calculated ECD curve of (10S)-1 obtained at the CAMB3LYP/def2SVP level matches well with the experimental CD spectrum (Figure 4).

Cochlearol G (2) has been previously described as a component of fruiting bodies of $G$. cochlear by Wang and co-workers (Wang et al., 2019a). Although the ROESY experiment performed by Wang et al. led to assignment of the relative configurations of the two stereogenic centers in the terminal six-member ring of 2 , the configuration of the
$\Delta^{3(4)}$ double bond remained unresolved. As a result, we carefully analyzed the ROESY spectrum and found the existence of a correlation $\mathrm{H}_{2}-2\left(\delta_{\mathrm{H}} 4.16\right) / \mathrm{H}_{2}-5\left(\delta_{\mathrm{H}} 2.65\right.$ and $2.42)$. This finding demonstrated that the $\Delta^{3(4)}$ double bond in 2 has $E$ configuration, rather than the $Z$ configuration depicted by Wang et al. (Wang et al., 2019a).

Ganodercin H (3) was isolated as a yellow gum. The HRESIMS of 3 gave a molecular ion $\mathrm{m} / \mathrm{z} 375.1804[\mathrm{M}+\mathrm{H}]^{+}$consistent with the molecular formula $\mathrm{C}_{21} \mathrm{H}_{26} \mathrm{O}_{6}$, indicating nine degrees of unsaturation. Detailed analysis of the $1 \mathrm{D}$ and 2D NMR data of 3 and ganotheaecoloid $\mathrm{D}$ shows that the $E \Delta^{3(4)}$-double bond is present in 3 rather than $Z$-configuration found in ganotheaecoloid $\mathrm{D}(\mathbf{8})$. This conclusion is supported by the ROESY correlation in 3 of H-2 $\left(\delta_{\mathrm{H}}\right.$ $4.09) / \mathrm{H}-5\left(\delta_{\mathrm{H}} 2.45\right)$. The relative configurations of their stereogenic centers in the terminal cyclohexane ring in 3 were assigned as $6 R^{\star}, 10 S^{\star}$ by using ROESY correlation of H-6 $\left(\delta_{\mathrm{H}} 1.94\right) / \mathrm{H}-10\left(\delta_{\mathrm{H}}\right.$ 3.38). Moreover, the absolute configurations at these centers were assigned as $6 R, 10 S$ by comparing its $\mathrm{CD}$ spectrum to that of ganotheaecoloid D.

Ganodercin I (4), isolated as a yellow gum, has the molecular formula $\mathrm{C}_{21} \mathrm{H}_{28} \mathrm{O}_{5}$ (eight degrees of unsaturation) based on HRESIMS analysis (m/z 361.2012 $[\mathrm{M}+\mathrm{H}]^{+}$; calcd for $\left.\mathrm{C}_{21} \mathrm{H}_{29} \mathrm{O}_{5}{ }^{+}, 361.2010\right)$. $1 \mathrm{D}$ and $2 \mathrm{D}$ NMR analysis shows that 4 has a structure that is similar to that of 3 . The presence of resonances associated with a terminal double bond $\left(\delta_{\mathrm{H}} 4.84\right.$, 
<smiles>CC1=C([C@H]2C=C(C(=O)O)[C@@H](C(=O)c3cc(O)ccc3O)CC2)[C@@]2(CCC1)CCCC2(O)O</smiles>

1<smiles>CC1=CC[C@]2(CCC3CCC(Cc4cc(O)ccc4O)=C3C(=O)O)C(C)=CC[C@@]12O</smiles>

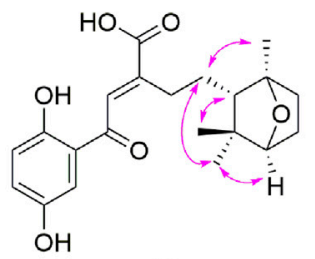

12<smiles>C=C1CC[C@]2(O)CC[C@]1([C@H]1C=C(C(=O)O)C(C(=O)c3cc(O)ccc3O)CC1)C2(C)C</smiles>

3<smiles>C=C1CCC(O)(O)[C@]1(C)CC[C@H](CC(=O)c1cc(O)ccc1O)C(=O)O</smiles>

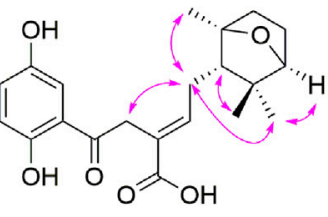

13

ROESY<smiles>C=C1CC[C@]2(O)C[C@]1(C)[C@@H]2CCC1CCC(Cc2cc(O)ccc2O)=C1C(=O)O</smiles><smiles>C=C1CC[C@@]2(O)C(=C)C[C@]1(CC[C@H](CC(=O)c1cc(O)ccc1O)C(=O)OC)C2(C)C</smiles>

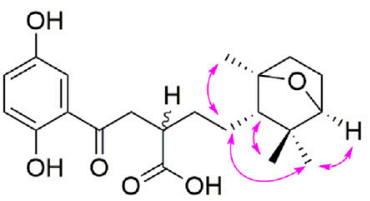

$14 / 15$
Ha-14, $\left.\delta_{\mathrm{H}} 4.61, \mathrm{Hb}-14\right)$ and two singlet methyls $\left(\delta_{\mathrm{H}} 0.97, \mathrm{H}_{3}-12\right.$, $\left.\delta_{\mathrm{H}} 0.64, \mathrm{H}_{3}-13\right)$ in ${ }^{1} \mathrm{H}$ NMR spectrum reveal that 4 contains the same terminal cyclohexane part that is present in 3 . The differences between $\mathbf{3}$ and $\mathbf{4}$ are that a methylene appears at $\mathrm{C}-1$ in the latter instead of a ketone in the former and the double bond $\Delta^{3(4)}$ of 3 is at $\Delta^{2(3)}$ in 4 . The ${ }^{1} \mathrm{H}^{-1} \mathrm{H}$ COSY correlation of $\mathrm{H}_{2}-1 \quad\left(\delta_{\mathrm{H}} 3.73\right.$ and 3.54$) / \mathrm{H}-2 \quad\left(\delta_{\mathrm{H}} 5.89\right)$ and the $\mathrm{HMBC}$ correlations of $\mathrm{H}-3^{\prime}\left(\delta_{\mathrm{H}} 6.58\right) / \mathrm{C}-1 \quad\left(\delta_{\mathrm{C}} 31.7\right), \mathrm{H}_{2}-1 / \mathrm{C}-3\left(\delta_{\mathrm{C}}\right.$ 133.6), $\mathrm{H}-2 / \mathrm{C}-15\left(\delta_{\mathrm{C}} 172.3\right)$ and $\mathrm{H}_{2}-4 / \mathrm{C}-2\left(\delta_{\mathrm{C}} 140.5\right), \mathrm{C}-3$, $\mathrm{C}-15$ support the above conclusions. The relative configurations of the two stereogenic centers in 4 were assigned as $6 R^{\star}, 10 S^{\star}$ based on ROESY correlation of H-6 $\left(\delta_{\mathrm{H}}\right.$ $1.66) / \mathrm{H}-10 \quad\left(\delta_{\mathrm{H}} 3.29\right)$. Further examination of ROESY correlation of $\mathrm{H}-2 / \mathrm{H}-4$ indicate that the $\Delta^{2(3)}$ double bond is $Z$-configuration. Finally, the experimental $\mathrm{CD}$ spectrum matched the calculated (CAM-B3LYP/def2SVP level) spectrum of $(6 R, 10 S)-4$.

Ganodercin J (5) has the same molecular formula as 4 (HRESIMS). Comariasons of ${ }^{1} \mathrm{H},{ }^{13} \mathrm{C} \mathrm{NMR}$, and DEPT spectra of 5 and $\mathbf{4}$ suggest they have similar structures. The difference between $\mathbf{5}$ and $\mathbf{4}$, inferred from 1D NMR data, is that the double bond on the terminal ring in 5 is at the $\Delta^{7(8)}$, whereas in compound 4 it is at $\Delta^{7(14)}$. This conclusion is supported by the observation of HMBC correlations of $\mathrm{H}_{3}-14\left(\delta_{\mathrm{H}} 1.70\right) / \mathrm{C}-6$ $\left(\delta_{\mathrm{C}} 50.2\right), \mathrm{C}-7\left(\delta_{\mathrm{C}} 137.8\right), \mathrm{C}-8\left(\delta_{\mathrm{C}} 120.1\right)$ and H-8 $\left(\delta_{\mathrm{H}} 5.22\right) / \mathrm{C}-6$. The configuration of the $\Delta^{2(3)}$ double bond was determined as $Z$ by observing a ROESY correlation of $\mathrm{H}-2 / \mathrm{H}_{2}-4$. Moreover, the observation of ROESY correlation of H-6 $\left(\delta_{\mathrm{H}} 1.66\right) / \mathrm{H}-10\left(\delta_{\mathrm{H}}\right.$ 3.36) indicated that 5 is the $6 R^{\star}, 10 S^{\star}$ diastereomer. The absolute configurations at the stereogenic centers in $\mathbf{5}$ were assigned as $6 R$ $10 S$ by comparing experimental $C D$ spectrum with calculated ECD curve (Figure 4).

Two isolated meroterpenoids 7 (ganodercin $\mathrm{K}$, yellow gum) and 8 (3-epi-ganodercin $\mathrm{K}$, yellow gum) were found to have the same molecular formula $\left(\mathrm{C}_{21} \mathrm{H}_{28} \mathrm{O}_{6}\right)$ and nearly identical NMR data, suggesting that they have the same planar structure with both lacking unsaturation in chains connecting the aryl and cyclohexane moieties. Indeed, a detailed comparison of their 1D NMR data with those of $\mathbf{1}$ shows that 7 and 8 lack the $\Delta^{3(4)}$ double bond in $\mathbf{1}$, a conclusion supported by ${ }^{1} \mathrm{H}-{ }^{1} \mathrm{H}$ COSY correlations of $\mathrm{H}-2 / \mathrm{H}-3 / \mathrm{H}-4 / \mathrm{H}-5$, and the HMBC correlations of H-2/C-1, C-3, C-4, C-15 and H-3/C-4, C-15. It was likely that 7 and 8 are diastereomers having $3 S^{\star}, 10 S^{*}$ and $3 R^{\star}, 10 S^{*}$ relative configurations at their stereogenic centers. Analysis of the CD spectra of $\mathbf{7}$ and $\mathbf{8}$ enabled the use of computational methods to elucidate the absolute configurations at their stereogenic centers. As seen by viewing Figure 4, the experimental CD spectrum of 7 well matches the calculated ECD curve of $3 R, 10 S$. In addition, the calculated ECD spectrum of $3 S, 10 S$ matches the experimental CD spectrum of $\mathbf{8}$.

Compounds $\mathbf{9}$ and $\mathbf{1 0}$ were previously isolated from $G$. theaecolum by Luo et al. (Luo et al., 2018) and characterized the enantiomers of ganotheaecoloid C. However, the absolute configurations at the three stereogenic centers in these substances were not determined in the earlier effort. As a result, we used TDDFT-ECD calculations to determine their absolute configurations. It can be seen from viewing Figure 4, that the experimental CD spectrum of $\mathbf{9}$ agrees well with the calculated 

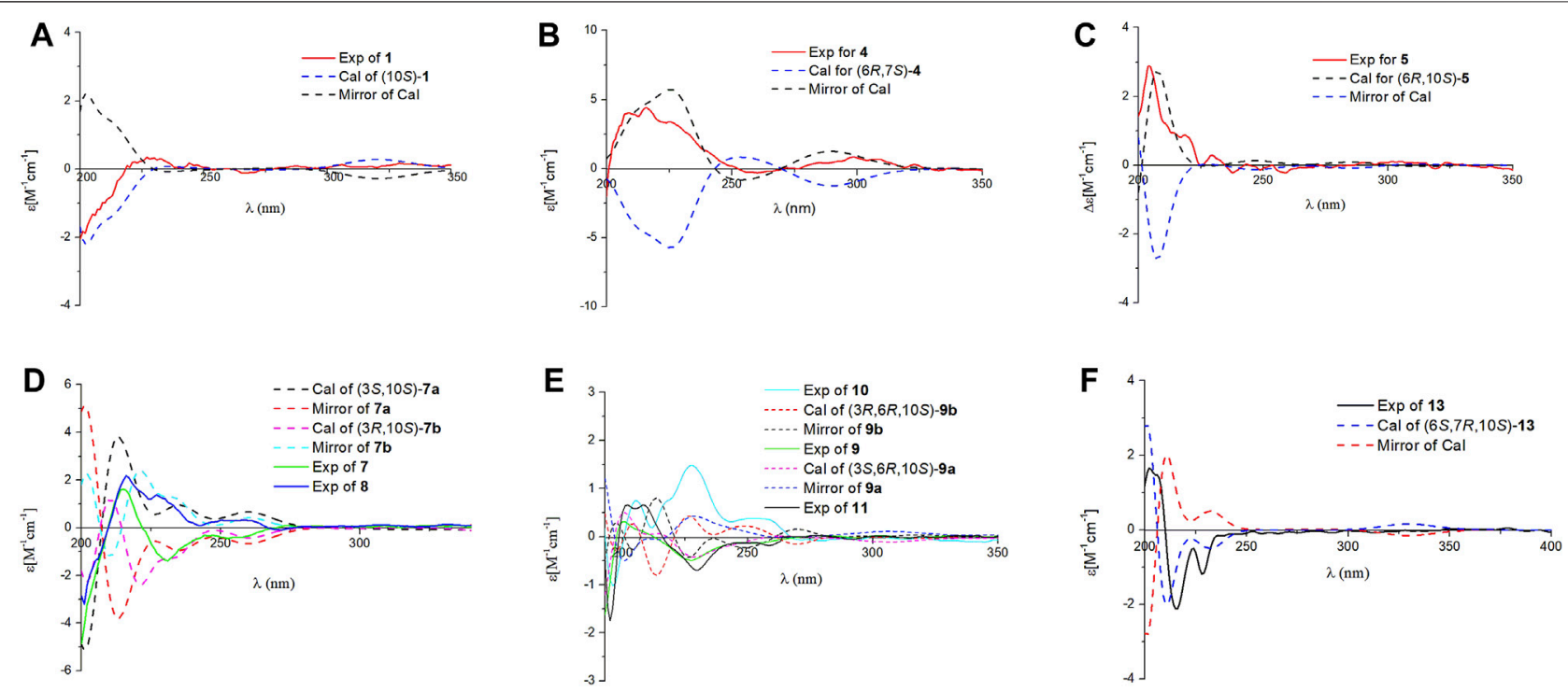

FIGURE 4 | Comparison of the calculated ECD and experimental CD spectra in MeOH. (A): The calculated ECD spectrum of (10S)-1 at CAM-B3LYP/def2SVP level, $\sigma=0.20 \mathrm{eV}$; shift $=-13 \mathrm{~nm}$. (B): The calculated ECD spectrum of (6R,10S)-4 at CAM-B3LYP/def2SVP level, $\sigma=0.28 \mathrm{eV}$; shift $=+8 \mathrm{~nm}$. (C): The calculated ECD spectrum of $(6 R, 10 S)-5$ at B3LYP/6-31G (d,p) level, $\sigma=0.25 \mathrm{eV}$; shift $=+20 \mathrm{~nm}$. (D): The calculated ECD spectrum of $(3 S, 10 S)-7 \mathbf{a}$ at B3LYP/6-31G (d,p) level, $\sigma=$ $0.25 \mathrm{eV}$; shift $=+20 \mathrm{~nm}$ and calculated ECD spectrum of $(3 R, 10 S)-7 \mathbf{b}$ at B3LYP/6-31G (d,p) level, $\sigma=0.23 \mathrm{eV}$; shift $=+20 \mathrm{~nm}$. (E): The calculated ECD spectrum of $(3 S, 6 R, 10 S)-9 \mathbf{a}$ at CAM-B3LYP/def2SVP level, $\sigma=0.28 \mathrm{eV}$; shift $=+13 \mathrm{~nm}$ and calculated ECD spectrum of $(3 R, 6 R, 10 S)-9 \mathbf{b}$ at B3LYP/6-31G (d,p) level, $\sigma=0.20 \mathrm{eV}$; shift $=+16 \mathrm{~nm}$. (F): The calculated ECD spectrum of $(6 S, 6 R, 10 S)-\mathbf{1 3}$ at CAM-B3LYP/def2SVP level, $\sigma=0.20 \mathrm{eV}$; shift $=-2 \mathrm{~nm}$.

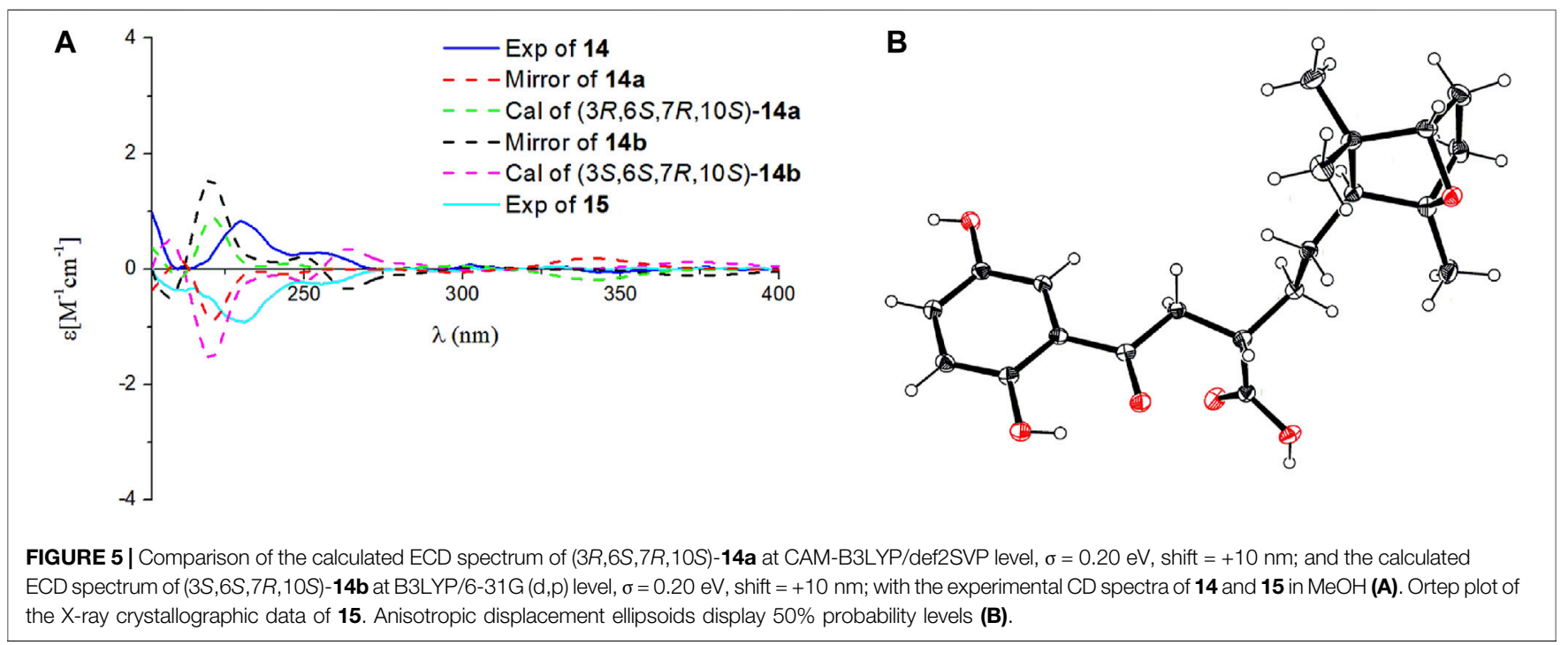

ECD curve of the $3 S, 6 R, 10 S$ stereoisomer. Importantly, the experimental CD spectrum of $\mathbf{1 0}$ is in close agreement with the calculated ECD spectrum of the $3 R, 6 R, 10 S$ stereoisomer, showing that $\mathbf{9}$ and $\mathbf{1 0}$ are actually epimers rather than enantiomers. By carefully analyzing the 1D NMR data reported by Luo, it was found that pairs of signals are present in the ${ }^{13} \mathrm{C}$ NMR spectrum, supporting our conclusion. The revised structures of $\mathbf{9}$ and $\mathbf{1 0}$ were renamed as ganodercin L for 9 and 3-epi-ganodercin L for $\mathbf{1 0 .}$

Ganodercin M (11) was obtained as a yellow gum and has the molecular formula $\mathrm{C}_{22} \mathrm{H}_{30} \mathrm{O}_{6}$ (HRESIMS ion observed at $\mathrm{m} / \mathrm{z}$
391.2120 $[\mathrm{M}+\mathrm{H}]^{+}$, calcd for 391.2115). The NMR data of $\mathbf{1 1}$ are similar to those of 9, except that the free C-15 carboxylic acid group in 9 is a methyl ester in $\mathbf{1 1}$. This conclusion is confirmed by observations of the $\mathrm{HMBC}$ correlation of $\mathrm{OCH}_{3} / \mathrm{C}-15$. The ROESY correlation of $\mathrm{H}-6 / \mathrm{H}-10$ showed that the relative configurations at the stereogenic centers in the terminal ring in 11 are $6 R^{\star}, 10 S^{*}$. The computational methods applied to 9 and 10 were used to determine the absolute configurations at the stereogenic centers in 11. Matching experimental and calculated CD spectra showed that $\mathbf{1 1}$ is the $3 S, 6 R, 10 S$ stereoisomer. 

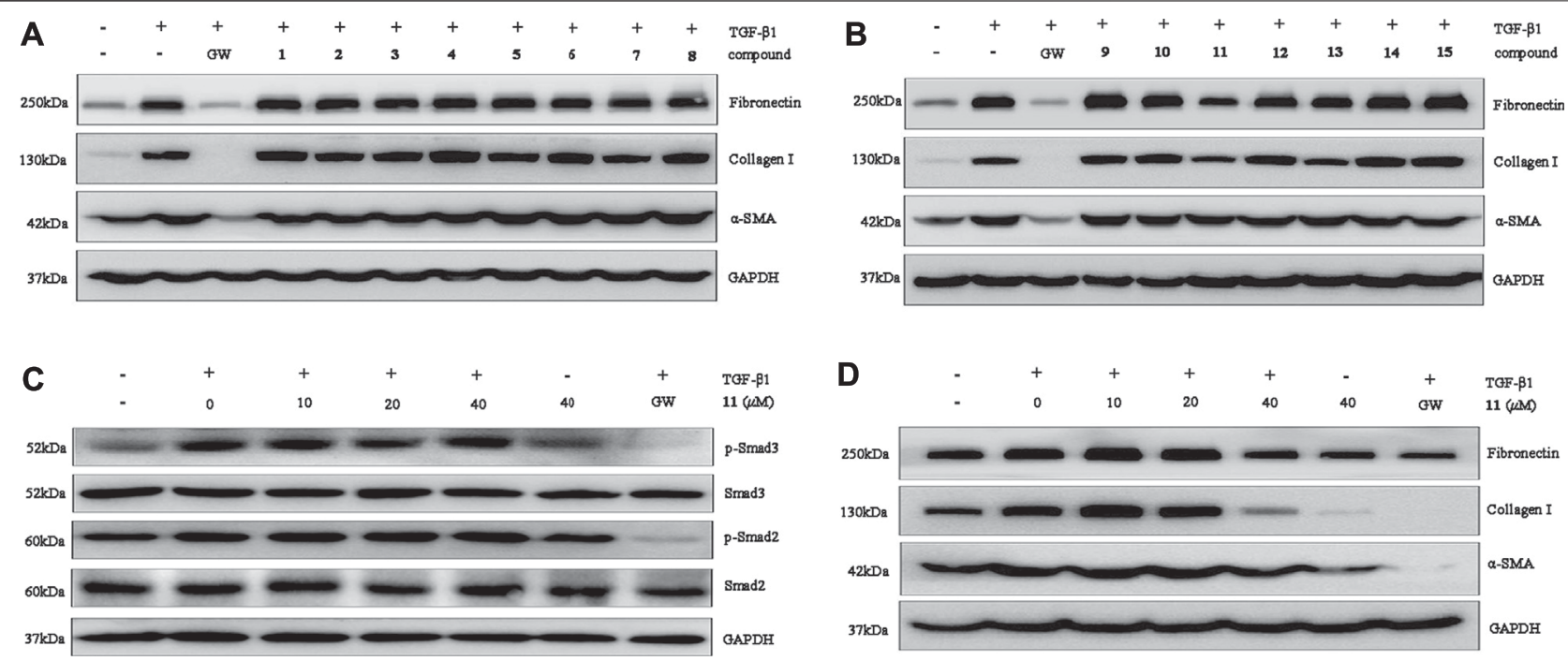

FIGURE 6 | Compounds 1-15 inhibit renal fibrosis in vitro. (A,B): Protein expression of collagen I, fibronectin, and $\alpha$-SMA was examined by Western blot analysis. (C): Compound $\mathbf{1 1}$ at $0 \mu \mathrm{M}, 10 \mu \mathrm{M}, 20 \mu \mathrm{M}$, and $40 \mu \mathrm{M}$ affected TGF- $\beta 1$-induced Smad phosphorylation. GAPDH was used as an internal control. Three independent experiments were performed. (D): Compound $\mathbf{1 1}$ dose-dependently inhibits fibronectin and collagen I expression. GAPDH was used as an internal control. GW was used as a positive control. GW: GW788388. Three independent experiments were performed.

The NMR spectroscopic data of ganodercin N (12) (yellow powder, $\left.\mathrm{C}_{21} \mathrm{H}_{26} \mathrm{O}_{6}\right)$ are similar to those of $(-)$-ganotheaecoloid F (Luo et al., 2018). One difference is that a methine $\left(\delta_{\mathrm{C}} 57.1\right)$ and a nonprotonated carbon $\left(\delta_{\mathrm{C}} 88.6\right)$ are present in 12 instead of the double bond $\left(\delta_{\mathrm{C}} 136.7\right.$ and $\delta_{\mathrm{C}}$ $128.6)$ in (-)-ganotheaecoloid $\mathrm{F}$. This proposal is supported by the HMBC correlations of $\mathrm{H}_{3}-14\left(\delta_{\mathrm{H}} 1.28\right) / \mathrm{C}-6\left(\delta_{\mathrm{C}} 57.1\right), \mathrm{C}-7$ $\left(\delta_{\mathrm{C}} 88.6\right), \mathrm{C}-8\left(\delta_{\mathrm{C}} 39.7\right)$. Also, the downfield chemical shift of $\mathrm{C}-10$ at $87.6 \mathrm{ppm}$, indicated that $\mathrm{C}-10$ is the oxygenated. Moreover, the HMBC correlation of $\mathrm{H}-10 / \mathrm{C}-7$ indicated that $\mathrm{C}-10$ is linked to $\mathrm{C}-7$ via an oxygen bridge. The relative configurations at the stereogenic centers in the terminal bicyclic ring in $\mathbf{1 2}$ were determined by using ROESY data. ROESY correlation of H-6 ( $\left.\delta_{\mathrm{H}} 1.26\right) / \mathrm{H}_{3}-12$ $\left(\delta_{\mathrm{H}}\right.$ 1.02) suggested that both $\mathrm{H}-6$ and $\mathrm{CH}_{3}-12$ have a $\beta$-orientation. Accordingly, the ROESY correlation of $\mathrm{H}-10$ $\left(\delta_{\mathrm{H}} 3.70\right) / \mathrm{H}_{3}-13\left(\delta_{\mathrm{H}} 0.97\right)$ revealed that $\mathrm{H}-10$ and $\mathrm{CH}_{3}-13$ have a $\alpha$-orientation. The $\mathrm{C}$ - 14 methyl was determined to have a $\alpha$-orientation, when consideration is given to the existence of the oxygen bridge. This proposal was further supported by the ROESY correlation of $\mathrm{H}_{2}-4\left(\delta_{\mathrm{H}} 2.58\right.$ and 2.52$) / \mathrm{H}_{3}-14$. Thus, the relative configurations at the stereogenic centers in 12 were assigned as $6 S^{\star}, 7 R^{\star}, 10 S^{\star}$. Since no strong correlation of $\mathrm{H}-2 / \mathrm{H}-4$ was observed, we hypothesized that the $\Delta^{2(3)}$ double bond in $\mathbf{1 2}$ is $E$. Density functional theory (DFT, B3LYP/6-311G(d,p) level) was used to verify this proposal. The experimental NMR data of $\mathbf{1 2}$ were compared with those calculated for 12a ( $E$-isomer) and 12b ( $Z$-isomer). It was found that the calculated ${ }^{13} \mathrm{C}$ NMR data for $12 \mathrm{a}$ possesses the highest $R^{2}$ value and a $100 \%$ probability in DP4+ analysis. Because no observable Cotton effects are present in the experimental CD spectrum of $\mathbf{1 2}$, it was not possible to use ECD calculations to assign stereochemistry. However, the absolute configuration at the stereogenic centers in $\mathbf{1 2}$ were assigned as $6 S, 7 R, 10 S$ by comparing the calculated specific optical rotation +22.2 for $(6 S, 7 R, 10 S)$ with the experimental one of $12\left([\alpha]_{\mathrm{D}}^{20}+22.5\right)$.

Ganodercin O (13) has the same molecular formula as $\mathbf{1 2}$ and similar NMR data, differing only by resonances associated with the location of the double bond. The position of double bond in $\mathbf{1 3}$ was demonstrated to be $\Delta^{3(4)}$ by the observation of ${ }^{1} \mathrm{H}-{ }^{1} \mathrm{H}$ COSY correlations of $\mathrm{H}$ 4/H-5/H-6, and the HMBC correlations of H-2/C-1, C-3, C-4, $\mathrm{C}-15$ and $\mathrm{H}-4 / \mathrm{C}-15$. The double bond $\Delta^{7(8)}$ was deduced to have the E-configuration by existence of the ROESY correlation of H-2/H-4. Similar to that in 12, H-6 and $\mathrm{CH}_{3}-12$ were determined to have a $\beta$-orientation by presence of the ROESY correlation of $\mathrm{H}-6 / \mathrm{H}_{3}-12$. Moreover, $\mathrm{H}-10$ and $\mathrm{CH}_{3}-14$ were assigned to have a $\alpha$-orientation by the ROESY correlations between $\mathrm{H}-10 / \mathrm{H}_{3}-13, \mathrm{H}_{2}-5 / \mathrm{H}-13, \mathrm{H}-14$. As a result, the relative configurations of the stereogenic centers in $\mathbf{1 3}$ were determined to be $6 S^{*}, 7 R^{\star}, 10 S^{*}$. Finally, a match between the computed [B3LYP/6-31 g(d,p)] ECD spectrum of $6 S, 7 R, 10 S-13$ and the experimental one provided the absolute configurations at the stereogenic centers in $\mathbf{1 3}$ (Figure 1).

Ganodercin P (14) and 3-epi-ganodercin P (15) were found to have the same molecular formula of $\mathrm{C}_{21} \mathrm{H}_{28} \mathrm{O}_{6}$ by using HRESIMS. Careful analysis revealed that their NMR data were similar, suggesting that they have the same planar structures. The observations of ${ }^{1} \mathrm{H}-{ }^{1} \mathrm{H}$ COSY correlations of $\mathrm{H}-2 / \mathrm{H}-3 / \mathrm{H}-4$, and the HMBC correlations of $\mathrm{H}-2 / \mathrm{C}-1, \mathrm{C}-15$ and $\mathrm{H}-3 / \mathrm{C}-15$ clearly revealed that one methylene $(\mathrm{C}-2)$ and one methine (C-3) in both 14 and 15 replace the $E$-double bond in 12. Because 14 and 15 contain the same terminal bicyclic ring that is present in $\mathbf{1 2}$ and $\mathbf{1 3}$, the relative configurations at the stereogenic centers were determined to be $6 S^{*}, 7 R^{\star}, 10 S^{*}$ by the presence of ROESY correlations $\mathrm{H}-6 / \mathrm{H}_{3}-12, \mathrm{H}-10 / \mathrm{H}_{3}-13, \mathrm{H}_{2}-5 /$ 


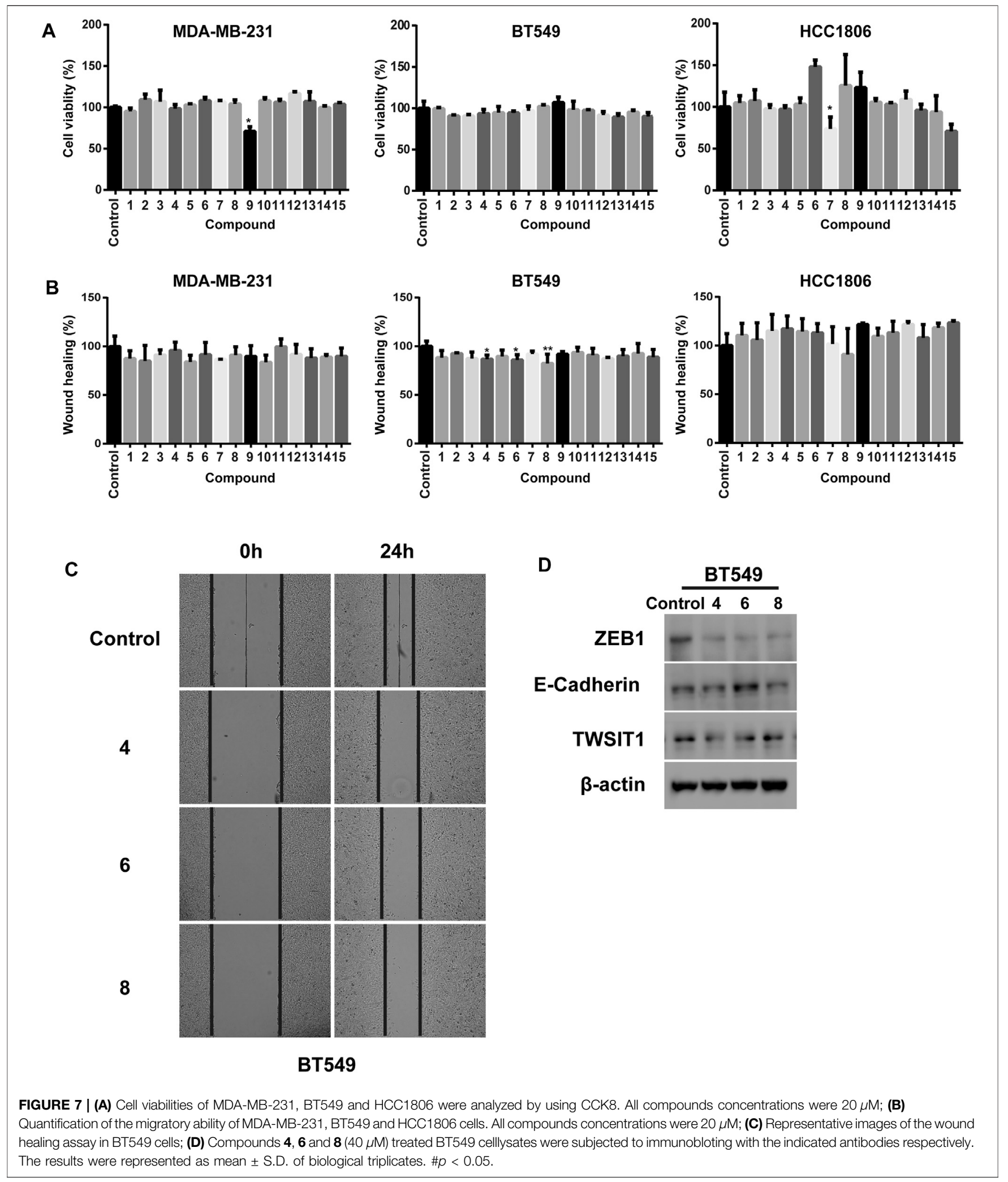

$\mathrm{H}_{3}-13, \mathrm{H}_{3}-14$. The relatively configuration of $\mathrm{C}-3$ is difficult to determined owing to the flexible nature of the chain. As a result, both 14 and 15 can be the two possible diastereomers $3 R^{\star}, 6 S^{\star}, 7 R^{\star}, 10 S^{*}$ and $3 S^{*}, 6 S^{*}, 7 R^{*}, 10 S^{*}$. Computational methods were used to determine the absolute configurations at the stereogenic centers in 14 and 15. The calculated curves of these substances showed that 
A

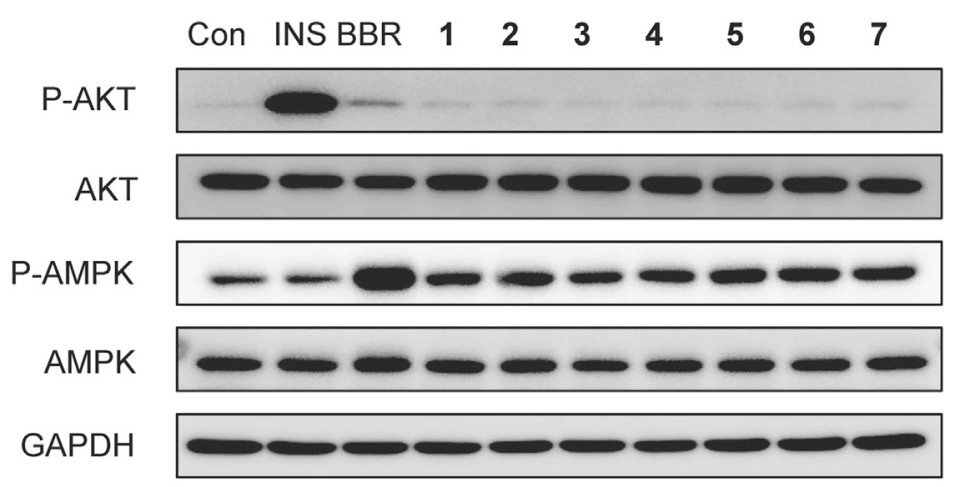

B

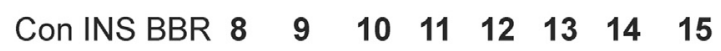

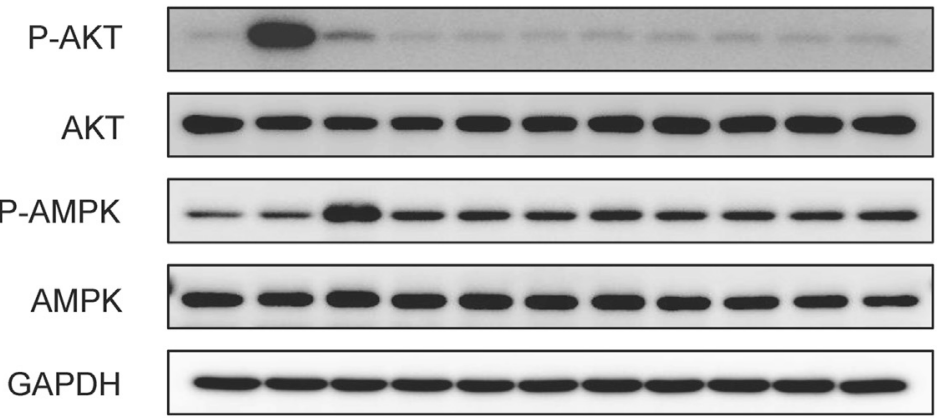

L6

C

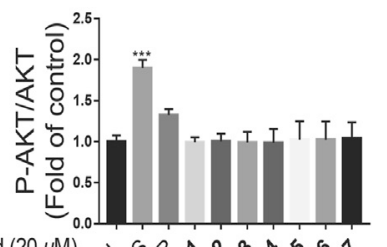

Compound $(20 \mu \mathrm{M})$

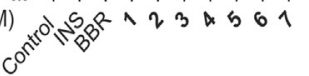

L6

D

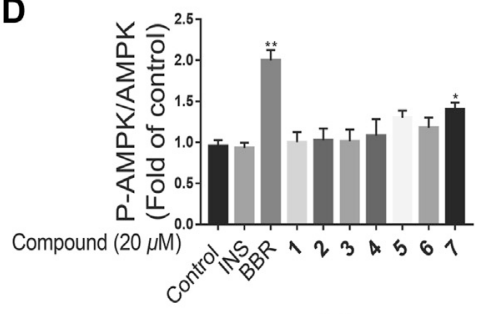

L6

$\mathbf{E}$

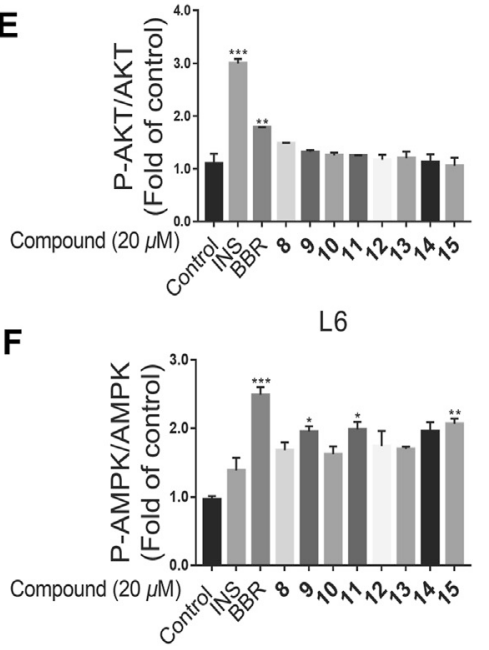

FIGURE 8 | The effects of compounds on L6 myotubes. (A-F): L6 myotubes were treated with vehicle or $20 \mu \mathrm{M}$ compounds or $10 \mu \mathrm{M} \mathrm{BBR} \mathrm{for} 24 \mathrm{~h}(n=3)$. Data are for three replicate experiments. $(n=3){ }^{*} p<0.05,{ }^{* *} p<0.01{ }^{* *} p<0.001$, one-way ANOVA.

the terminal oxygen bridge doe not contribute to the Cotton effects (Figure 5). As a result, the absolute configuration of C-3 in 14 is $R$ and that of compound $\mathbf{1 5}$ is $S$.

X-ray crustallographic analysis of $\mathbf{1 5}$ was carried out, which was generated by crystallization from Kappa single diffractometer in $\mathrm{CuK} \alpha$ with a Flack parameter of -0.08 (7). Analysis of the crystal data leads to assignment of the absolute configurations at the stereogenic centers in $\mathbf{1 5}$ to be $3 S, 6 S, 7 R, 10 S$. Since $\mathbf{1 4}$ and $\mathbf{1 5}$ are C-3 epimers, the absolute configurations at the centers in $\mathbf{1 4}$ are $3 R, 6 S, 7 R, 10 S$.

The final substance isolated was (-)-ganotheaecoloid F (6), which was identified by comparison its spectroscopic data with those reported in the literature (Luo et al., 2018).

In this study, we have determined the structures and absolute configurations at the stereogenic centers in 15 meroterpenoids isolated from dried fruiting bodies of $G$. cochlear. It is worthy noting that all of these substances contain a terminal cyclohexane ring and all are enantiomerically pure. Meroterpenoids of this type isolated from Ganoderma have been also found to exist in enantiomerically pure (Luo et al., 2018; Wang et al., 2019a; Wang et al., 2019b). Among them, the absolute configuration of four structures was successfully determined. The absolute configurations at the centers in these substances as well as the fact that C-10 in all have the $S$-configuration might be related to their biosynthetic origin, and might aid subsequent structural identification of analogues.

The protective activity of the meroterpenoids isolated in this effort were assessed by observing the expression of renal fibroblast biomarkers in TGF- $\beta 1$-induced NRK-52E, which plays an important role in stimulation of renal fibroblasts (Zhang et al., 2012; Meng et al., 2015). The results showed that 11 displays selective inhibitory activity in that it significantly inhibits over-expression of fibronectin, collagen I and $\alpha$-SMA 
at the protein level at a concentration of $40 \mu \mathrm{M}$ (Figures 6A,B). In addition, western blot analysis, carried out in a dose-concentration dependent manner, demonstrated that the optimum activity of 11 is $40 \mu \mathrm{M}$ (Figure 6D). To explore the mechanism underlying the antifibrotic effect of, Smad2/3 phosphorylation was investigated. We found that 11 has no effect on phosphorylation of Smad2 or Smad3 in TGF$\beta 1$-induced NRK-52E cells at concentrations of $10 \mu \mathrm{M}, 20$ and $40 \mu \mathrm{M}$ (Figure 6C), suggesting that its effects could play potential roles in renal fibrosis through a non-Smad pathway.

We investigated the cellular phenotype promoted by the isolated substances in breast cancer cells by using a cell viability and a wound healing assay in three TNBC cell lines including MDA-MB-231, BT549 and HCC1806. Inspection of the plots given in Figure 7A shows that treatment with $\mathbf{7}$ and $\mathbf{9}$ results in significant decreases in cell viability of 29.2 and $26.3 \%$ in MDA-MB-231 and HCC1806 cells, respectively. The other meroterpenoids have negligible inhibitory effects on cell viability even at concentrations as high as $20 \mu \mathrm{M}$ (Figure 7A). Interestingly, although all the fifteen substances have rather low cytotoxicities toward these three breast cancer cell lines, three of the isolates including 4, 6 and 8 significant inhibit the migration ability of BT549 cells. All of the other meroterpenoids display no significant effects on MDA-MB-231 and HCC1806 cells (Figures 7B,C). Moreover, we observed that treatment with 4 and 8 markedly decreases the protein level of TWIST1 and ZEB1 without noticeably affecting the E-cadherin level in BT549 cell lysates. Meroterpenoid 6 not only decreases the protein level of TWIST1 and ZEB1, it also increases the protein level of E-cadherin (Figure 7D). ZEB1, E-cadherin and TWIST1 are generally acknowledged to be transcriptional factors driving the Epithelial-Mesenchymal Transition (EMT), one of the most important pathogenic events occurring in the initiation of cancer metastasis. Thus, the data indicate that 4, 6 and 8 suppress the metastatic potential of TNBC cells through down regulation of EMT, and consequently they are promising lead compounds for the development of the anti-cancer drugs against metastasis of TNBC.

All the isolated meroterpenoids at a concentration of $20 \mu \mathrm{M}$ were exposed to normal L6 myotubes cells for $24 \mathrm{~h}$. The control group was treated with insulin (INS, $100 \mathrm{nM}, 15 \mathrm{~min}$ ) and Berberine (BBR, $10 \mu \mathrm{M}, 24 \mathrm{~h})$. Notably, INS and BBR treatment led to significant increases in the phosphorylation AKT and AMPK, respectively. Compared with the control group, the meroterpenoids have no significant impact on the AKT pathway. Meanwhile, 7, 9, 11 and 15 significantly upregulate $\mathrm{p}$-AMPK protein expression (Figure 8). This finding suggests that these substances might enhance insulin sensitivity by activating AMP-activated protein kinase (AMPK) in normal L6 myotubes cells.

\section{REFERENCES}

Collignon, J., Lousberg, L., Schroeder, H., and Jerusalem, G. (2016). Triple Negative Breast Cancer: Treatment Challenges and Solutions. Breast Cancer (Dove Med. Press.) 8, 93-107. doi:10.2147/BCTT.S69488

El Dine, R. S., El Halawany, A. M., Ma, C. M., and Hattori, M. (2009). Inhibition of the Dimerization and Active Site of HIV-1 Protease by Secondary Metabolites

\section{CONCLUSION}

In conclusion, the study described above resulted in the isolation of eleven new meroterpenoids, and four known meroterpenoids. The structure of cochlearol G (2) was revised, and the absolute configurations at the stereogenic centers in $\mathbf{9}$ and $\mathbf{1 0}$ were determined by using ECD calculations. Biological studies related to renal fibrosis showed that (1) 11 inhibits over-expression of fibronectin, collagen I and $\alpha$-SMA, (2) 4, 6 and 8 significantly inhibitof the migration ability of BT549 cells, (3) 6 decreases the protein level of TWIST1 and ZEB1 and increases the protein level of E-cadherin, and (4) 7, 9, 11 and 15 significantly up-regulate p-AMPK protein expression in normal L6 myotubes cells.

\section{DATA AVAILABILITY STATEMENT}

The original contributions presented in the study are included in the article/Supplementary Material, further inquiries can be directed to the corresponding authors.

\section{AUTHOR CONTRIBUTIONS}

YC designed the research. FQ conducted chemical experiments. TX, YL, and DC conducted biological experiments in vitro. FQ, TX, HZ, LL, and YC analyzed data. FQ and YC wrote and revised the manuscript. All authors discussed the results and commented on the manuscript at all stages.

\section{FUNDING}

We are indebted to the NSFC-Joint Foundation of Yunnan Province (U1702287), National Natural Science Foundation of China (82030115), Shenzhen Fundamental Research Program (JCYJ20200109113803838), National Science Fund for Distinguished Young Scholars (81525026), Guangdong Key Laboratory for Functional Substances in Medicinal Edible Resources and Healthcare Products (2021B1212040015), and National Natural Science Foundation of China (82104036).

\section{SUPPLEMENTARY MATERIAL}

The Supplementary Material for this article can be found online at: https://www.frontiersin.org/articles/10.3389/fchem.2021.783705/ full\#supplementary-material

from the Vietnamese Mushroom Ganoderma colossum. J. Nat. Prod. 72, 2019-2023. doi: $10.1021 / \mathrm{np} 900279 \mathrm{u}$

Jiang, M., Wu, Z., Liu, L., and Chen, S. (2021). The Chemistry and Biology of Fungal Meroterpenoids (2009-2019). Org. Biomol. Chem. 19, 1644-1704. doi:10.1039/d0ob02162h

Kumar, A. (2021). Ganoderma lucidum: a Traditional Chinese Medicine Used for Curing Tumors. Int. J. Pharm. Pharm. Sci. 13, 1-13. doi:10.22159/ ijpps.2021v13i3.40614 
Luo, Q., Tu, Z. C., Yang, Z. L., and Cheng, Y. X. (2018). Meroterpenoids from the Fruiting Bodies of Ganoderma theaecolum. Fitoterapia 125, 273-280. doi:10.1016/j.fitote.2018.01.015

Luo, Q., Wang, X. L., Di, L., Yan, Y. M., Lu, Q., Yang, X. H., et al. (2015). Isolation and Identification of Renoprotective Substances from the Mushroom Ganoderma lucidum. Tetrahedron 71, 840-845. doi:10.1016/j.tet.2014.12.052

Meng, X. H., Qin, F. Y., Jiang, X. T., Li, Y., and Cheng, Y. X. (2021). $( \pm)$-Gancochlearols J - N, Renoprotective Meroterpenoids from Ganoderma cochlear. Bioorg. Chem. 112, 104950. doi:10.1016/j.bioorg.2021.104950

Meng, X. M., Tang, P. M. K., Li, J., and Lan, H. Y. (2015). TGF- $\beta /$ Smad Signaling in Renal Fibrosis. Front. Physiol. 6, 82. doi:10.3389/fphys.2015.00082

Wang, K., Bao, L., Ma, K., Zhang, J., Chen, B., Han, J., et al. (2017). A Novel Class of $\alpha$-glucosidase and HMG-CoA Reductase Inhibitors from Ganoderma leucocontextum and the Anti-diabetic Properties of Ganomycin I in KK-Ay Mice. Eur. J. Med. Chem. 127, 1035-1046. doi:10.1016/j.ejmech.2016.11.015

Wang, L., Li, J. Q., Zhang, J., Li, Z. M., Liu, H. G., and Wang, Y. Z. (2020). Traditional Uses, Chemical Components and Pharmacological Activities of the Genus Ganoderma P. Karst.: a Review. RSC Adv. 10, 42084-42097. doi:10.1039/d0ra07219b

Wang, X. L., Wu, Z. H., Di, L., Zhou, F. J., Yan, Y. M., and Cheng, Y. X. (2019a). Renoprotective Meroterpenoids from the Fungus Ganoderma cochlear. Fitoterapia 132, 88-93. doi:10.1016/j.fitote.2018.12.002

Wang, X. L., Wu, Z. H., Di, L., Zhou, F. J., Yan, Y. M., and Cheng, Y. X. (2019b). Renoprotective Phenolic Meroterpenoids from the Mushroom Ganoderma cochlear. Phytochemistry 162, 199-206. doi:10.1016/ j.phytochem.2019.03.019
Yan, Y. M., Wang, X. L., Luo, Q., Jiang, L. P., Yang, C. P., Hou, B., et al. (2015). Metabolites from the Mushroom Ganoderma Lingzhi as Stimulators of Neural Stem Cell Proliferation. Phytochemistry 114, 155-162. doi:10.1016/ j.phytochem.2015.03.013

Zhang, W., Tsuda, M., Yang, G. X., Tsuneyama, K., He, X. S., Ansari, A. A., et al. (2012). Lymphoma-like T Cell Infiltration in Liver Is Associated with Increased Copy Number of Dominant Negative Form of TGF $\beta$ Receptor II. PLoS One 7, e49413. doi:10.1371/journal.pone.0049413

Conflict of Interest: The authors declare that the research was conducted in the absence of any commercial or financial relationships that could be construed as a potential conflict of interest.

Publisher's Note: All claims expressed in this article are solely those of the authors and do not necessarily represent those of their affiliated organizations, or those of the publisher, the editors and the reviewers. Any product that may be evaluated in this article, or claim that may be made by its manufacturer, is not guaranteed or endorsed by the publisher.

Copyright (c) 2021 Qin, Xu, Li, Zhang, Cai, Liu and Cheng. This is an open-access article distributed under the terms of the Creative Commons Attribution License (CC $B Y)$. The use, distribution or reproduction in other forums is permitted, provided the original author(s) and the copyright owner(s) are credited and that the original publication in this journal is cited, in accordance with accepted academic practice. No use, distribution or reproduction is permitted which does not comply with these terms. 